\title{
ON THE DISTRIBUTION OF THE NEARLY UNSTABLE AR(1) PROCESS WITH HEAVY TAILS
}

\author{
MARIANA OLVERA-CRAVIOTO, ${ }^{*}$ Columbia University
}

\begin{abstract}
We consider a nearly unstable, or near unit root, AR(1) process with regularly varying innovations. Two different approximations for the stationary distribution of such processes exist: a Gaussian approximation arising from the nearly unstable nature of the process and a heavy-tail approximation related to the tail asymptotics of the innovations. We combine these two approximations to obtain a new uniform approximation that is valid on the entire real line. As a corollary, we obtain a precise description of the regions where each of the Gaussian and heavy-tail approximations should be used.
\end{abstract}

Keywords: AR process; near unit root; heavy tail; weighted sum; uniform approximation; large deviation

2000 Mathematics Subject Classification: Primary 41A60; 37M10

Secondary 60F10; 60G10; 60G50; 60G70

$62 \mathrm{E} 17$

\section{Introduction}

First-order autoregressive processes are found in a variety of settings, ranging from the biological and natural sciences to financial and insurance applications. The analysis of such processes has long been a topic of great interest in the time series community, where considerable literature exists about the asymptotic properties of estimators for the regression parameter and the corresponding hypothesis tests. From an applications perspective, one often seeks conditions that would allow simple approximations for the distribution of these processes. We consider two such conditions here: a regime that allows us to obtain a Gaussian approximation and a condition on the distribution of the innovations that provides asymptotics for the extreme tail distribution.

When the regression parameter is close to one, the first-order autoregressive process is known as a nearly unstable (nonstationary) $\mathrm{AR}(1)$ process, sometimes also referred to as a process with near unit root. Similar to the study of queues in heavy traffic, this asymptotic regime can be used to derive approximations for the distribution of the process. One such approximation, due to Cumberland and Sykes [11], states that the distribution of the nearly unstable AR(1) process $\left\{Z_{n}: n \geq 0\right\}$, given by $Z_{n+1}=\rho Z_{n}+\xi_{n+1}, Z_{0}=z, Z_{0}=z$ and $|\rho|$ close to 1 , can be approximated by an Ornstein-Uhlenbeck process, provided that $\mathrm{E}\left[\xi_{1}\right]=0, \sigma^{2}=\mathrm{E}\left[\xi_{1}^{2}\right]$, and $\mathrm{E}\left[\xi_{1}^{4}\right]<\infty$. This result suggests the following approximation for its steady-state distribution:

$$
Z_{\infty} \stackrel{\text { D }}{\approx} \frac{\sigma}{\sqrt{2(1-\rho)}} Z
$$

where $Z$ is a standard normal random variable.

Received 4 November 2008; revision received 11 December 2009.

* Postal address: Department of Industrial Engineering and Operations Research, Columbia University, 306 S. W. Mudd Building, 500 W. 120th Street, New York, NY 10027, USA. Email address: molvera@ieor.columbia.edu 
A different set of results on general autoregressive processes (not only the AR(1) process) considers the special case where the innovations $\left(\xi_{i}: i \geq 0\right)$ belong to some kind of heavy-tail distribution (e.g. regularly varying). Typically, in the presence of heavy tails, it is possible to obtain asymptotic expressions for the tail distribution of sums of random variables. Such asymptotics are often tractable even in the more general setting of autoregressive processes, and a considerable amount of literature exists in this context (see the comments at the end of Section 2). Here we consider the case where the innovations belong to a regularly varying distribution with index $\alpha>2$, for which the following asymptotic is known to hold for $0<$ $\rho<1$ :

$$
\mathrm{P}\left(Z_{\infty}>x\right) \sim \mathrm{P}\left(\xi_{1}>x\right) \sum_{n=0}^{\infty} \rho^{\alpha n}=\left(1-\rho^{\alpha}\right)^{-1} \mathrm{P}\left(\xi_{1}>x\right), \quad x \rightarrow \infty
$$

(see [16, Lemma A.3] for a more general result).

Both approximations (1.1) and (1.2) apply to the model we consider here, the nearly unstable $\mathrm{AR}(1)$ process with regularly varying innovations. Nonetheless, the two are very different in nature, with the former providing approximations for moderate values of the tail and the latter for values in the extreme tail. The main result we present gives an approximation that is uniformly good on the entire real line, making it unnecessary to determine for each value of $x$ which approximation is appropriate. Moreover, we can easily determine from our uniform approximation the exact point at which the behavior of $Z_{\infty}$ transitions from the Gaussian approximation into the heavy-tail asymptotic, giving a precise notion of where each of approximations (1.1) and (1.2) are valid.

The author has been informed through personal communication that a similar paper by José Blanchet is in preparation. This paper discusses the more general AR $(k)$ model and will provide higher-order terms for the region where the Gaussian approximation is valid.

\section{Main results}

We consider a first-order autoregressive process of the form

$$
Z_{n+1}=\rho Z_{n}+\xi_{n+1}, \quad Z_{0}=z
$$

where $\left(\xi_{i}: i \geq 0\right)$ is a sequence of mean 0 independent and identically distributed random variables having distribution $F$, and $z \in \mathbb{R}$. It is well known that, provided $|\rho|<1$, the process $\left(Z_{n}: n \geq 0\right)$ possesses a stationary distribution. In particular, by iterating (2.1) we obtain

$$
Z_{n}=\rho^{n} z+\sum_{i=0}^{n-1} \rho^{i} \xi_{n-i}
$$

from which it follows that if $Z_{\infty}$ is a stationary version of the process then

$$
Z_{\infty} \stackrel{\mathrm{D}}{=} \sum_{i=0}^{\infty} \rho^{i} \xi_{i}
$$

where ' $\stackrel{D}{=}$ denotes equality in distribution. When $\rho$ is close to 1 , the process is said to be nearly unstable (nonstationary), and in this setting the distribution of the process can be analyzed by 
using a continuous time-scaled version of the process. Define $\left(Y_{m}(t): t \geq 0\right)$ according to $Y_{m}(0)=0$,

$$
\begin{gathered}
Y_{m}\left(\frac{i}{m}\right)=\left(1-\frac{1}{m}\right) Y_{m}\left(\frac{i-1}{m}\right)+\frac{\xi_{i}}{\sqrt{m}}, \quad i=1,2, \ldots, \\
Y_{m}(t)=Y_{m}\left(\frac{i}{m}\right) \quad \text { for } \frac{i}{m} \leq t<\frac{i+1}{m}
\end{gathered}
$$

By setting $\rho=1-1 / m$ and $Z_{n}=\sqrt{m} Y_{m}(n / m)$, we can recover the discrete-time process

$$
Z_{n}=\rho Z_{n-1}+\xi_{n}, \quad Z_{0}=0 .
$$

It was proven in [11] that if $\mathrm{E}\left[\xi_{1}\right]=0, \mathrm{E}\left[\xi_{1}^{2}\right]=\sigma^{2}$, and $\mathrm{E}\left[\xi_{1}^{4}\right]<\infty$, then, as $m \rightarrow \infty, Y_{m}(t)$ converges in distribution to $Y(t)$, where $Y(t)$ is a zero-mean Ornstein-Uhlenbeck process satisfying $Y(0)=0$, and

$$
\mathrm{d} Y(t)=-Y(t) \mathrm{d} t+\sigma \mathrm{d} B(t) .
$$

This result suggests the approximation

$$
\mathrm{P}\left(Z_{\infty}>x\right) \approx \mathrm{P}\left((1-\rho)^{-1 / 2} Y(\infty)>x\right)=1-\Phi\left(\frac{x \sqrt{2(1-\rho)}}{\sigma}\right),
$$

where $\Phi(t)=\int_{-\infty}^{t} \phi(u) \mathrm{d} u$ is the standard normal distribution function. This same approximation for $Z_{\infty}$ can alternatively be derived via Lindeberg's theorem (see [3, p. 357]). This nearly unstable AR(1) process has received considerable attention since the mid 1970s, both in the time series literature as well as the econometrics literature (see, for example, [6], [10], [24], and the references therein).

When the innovations $\left(\xi_{i}: i \geq 1\right)$ are heavy tailed, say regularly varying, then a different kind of approximation is available for the tail distribution of $Z_{\infty}$. In particular, under the so-called balance condition

$$
\bar{F}(t)=p t^{-\alpha} L(t) \quad \text { and } \quad F(-t) \sim q t^{-\alpha} L(t) \quad \text { as } t \rightarrow \infty
$$

for some $p \in(0,1]$ and $q=1-p$ (by $f(t) \sim g(t)$ we mean that $\lim _{t \rightarrow \infty} f(t) / g(t)=1$ ), we can obtain the asymptotic equivalence when $0<\rho<1$ :

$$
\mathrm{P}\left(Z_{\infty}>x\right) \sim \bar{F}(x) \sum_{n=0}^{\infty} \rho^{\alpha n}, \quad x \rightarrow \infty .
$$

More generally, if we consider a weighted infinite series of the form $Y=\sum_{i=0}^{\infty} \psi_{i} \xi_{i}$, when the innovations are regularly varying with index $\alpha>0$, the following asymptotic is known to hold under certain conditions on the coefficients $\left(\psi_{i}: i \geq 0\right)$ :

$$
\mathrm{P}(Y>x) \sim \mathrm{P}\left(\left|\xi_{1}\right|>x\right) \sum_{i=0}^{\infty}\left[p\left(\psi_{i}^{+}\right)^{\alpha}+q\left(\psi_{i}^{-}\right)^{\alpha}\right], \quad x \rightarrow \infty .
$$

Early results in this direction are attributed to Cline [8], [9] (for the case $0<\alpha \leq 1$ ) and can be found in [21, p. 226]. Best possible conditions on the coefficients are given in [16], where in particular we find that, when $\alpha>2$ and $\mathrm{E}\left[\xi_{1}\right]=0,(2.5)$ holds provided that $\sum_{i=0}^{\infty} \psi_{i}^{2}<\infty$. 
We refer the reader to [15] for a more complete compilation of results related to sums, series, and products of regularly varying functions, and to [4] for a thorough treatment of regularly varying functions and their properties.

In terms of the nearly unstable AR(1) process we are considering here, both (2.2) and (2.4) provide approximations for the tail distribution of $Z_{\infty}$. Clearly, as a function of $x$, the normal approximation predicts a tail that decreases superexponentially as $x \rightarrow \infty$ (since $1-\Phi(t) \sim$ $\phi(t) / t$, while the heavy-tailed asymptotic predicts a regularly varying tail. This apparent inconsistency can be explained by observing that the regions for which each approximation is valid are not the same; the normal approximation is known to be good for values of $x$ of order $(1-\rho)^{-1 / 2}$, while the heavy-tailed asymptotic refers to values of $x$ in the extreme tail $\left(x \gg(1-\rho)^{-1 / 2}\right)$. To give us a better idea of where the transition between the two different behaviors occurs, we can solve (approximately) for the value of $x$ for which (2.2) and (2.4) are equal, that is,

$$
-\frac{x^{2}(1-\rho)}{\sigma^{2}}-\log \left[x(1-\rho)^{-1 / 2}\right] \approx-\alpha \log x+\log L(x)+\log \left((1-\rho)^{-1}\right) .
$$

It follows from here that the solution $x^{*}(\rho)$ to this equation satisfies

$$
x^{*}(\rho) \sim \kappa(1-\rho)^{-1 / 2}|\log (1-\rho)|^{1 / 2}
$$

as $\rho \nearrow 1$, where $\kappa=\sigma \sqrt{(\alpha-2) / 2}$. This heuristic analysis can be done rigorously, and Theorem 2.1, below, gives a precise statement of our result. Similar results in the context of the $\mathrm{M} / \mathrm{G} / 1$ queue were obtained in [19] and [18]. A careful analysis of the probability $\mathrm{P}\left(Z_{\infty}>x\right)$ for values of $x$ close to this threshold allows us to provide an approximation that is uniform across all values of $x$, from where we can determine the exact regions over which each of the two approximations holds.

In the statements of the theorems given below and throughout the rest of the paper, we always assume the following.

Assumption 2.1. ( $\left.X_{i}: i \geq 0\right)$ is a sequence of independent and identically distributed random variables with common distribution $F$, where $F$ satisfies the balance condition (2.3) with $\alpha>2$ and $L(\cdot)$ is a slowly varying function. Furthermore, we assume that $\mathrm{E}\left[X_{1}\right]=0$ and set $\sigma^{2}=\mathrm{E}\left[X_{1}^{2}\right]$.

Given the relation $Z_{\infty} \stackrel{\mathrm{D}}{=} \sum_{n=0}^{\infty} \rho^{n} X_{n}$, we can consider directly the series $Y_{\infty}=\sum_{n=0}^{\infty} \rho^{n} X_{n}$ in the statements of the results.

Theorem 2.1. Let $Y_{\infty}=\sum_{n=0}^{\infty} \rho^{n} X_{n}$. Then, as $\rho \nearrow 1$,

$$
\sup _{x \in \mathbb{R}}\left|\frac{\mathrm{P}\left(Y_{\infty}>x\right)}{\Phi\left(-x \sqrt{1-\rho^{2}} / \sigma\right)+1\left(x \geq(1-\rho)^{-1 / 2}\right)\left(1-\rho^{\alpha}\right)^{-1} \bar{F}(x)}-1\right| \rightarrow 0 .
$$

Remark 2.1. Using Lemma 3.3 of [19], we can prove that the same asymptotic holds uniformly for $0<\rho<1$ as $x \rightarrow \infty$.

As a consequence of the uniform limit, we can obtain a very precise description of the threshold at which the behavior of $Y_{\infty}$ transitions from the normal approximation into the heavy-tailed asymptotic. 
Corollary 2.1. Define $z(\rho)=\kappa(1-\rho)^{-1 / 2}|\log (1-\rho)|^{1 / 2}$, where $\kappa=\sigma \sqrt{(\alpha-2) / 2}$. Then the following assertions hold.

(a) For any $c<1$, as $\rho \nearrow 1$,

$$
\sup _{x \leq c z(\rho)}\left|\frac{\mathrm{P}\left(Y_{\infty}>x\right)}{\Phi\left(-x \sqrt{1-\rho^{2}} / \sigma\right)}-1\right| \rightarrow 0
$$

Furthermore, if $c=1,(2.6)$ holds provided that $\sup _{0 \leq t \leq x} L(t) /(\log x)^{(\alpha-1) / 2} \rightarrow 0$.

(b) For any $c>1$, as $\rho \nearrow 1$,

$$
\sup _{x \geq c z(\rho)}\left|\frac{\mathrm{P}\left(Y_{\infty}>x\right)}{\left(1-\rho^{\alpha}\right)^{-1} \bar{F}(x)}-1\right| \rightarrow 0
$$

Furthermore, if $c=1$, (2.7) holds provided that $\inf _{t \geq x} L(t) /(\log x)^{(\alpha-1) / 2} \rightarrow \infty$.

The main idea of the proof of Theorem 2.1 is to decompose $Y_{\infty}$ into two pieces, the first one with a finite number of summands and the second one corresponding to the tail of the infinite series. The analysis of the finite sum $Y_{K}=\sum_{n=0}^{K} \rho^{n} X_{n}$ can be done following the same lines as the analysis of the random walk with heavy-tailed increments (see [5] for an extensive study of such results, including the partial sums with nonidentically distributed increments). In particular, the style of the proofs we give for the region where both the normal approximation and the heavy-tailed asymptotic play a role, resembles in spirit the work done by Rozovskiî [23] in the random walk setting.

Theorems 2.2 and 2.3, below, give the asymptotic behavior of the partial sums $Y_{K}$ for moderate and large values of $x$, respectively. The results we present below for the partial sums are of potential interest in their own right, which is why the thresholds are given in terms of a quantity related to the variance of $Y_{K}$ (note that $\operatorname{var}\left(Y_{K}\right)=\sigma^{2} S_{K}(\rho)$ ). The condition that $K \rightarrow \infty$ in Theorem 2.2 is the minimum condition to guarantee that $S_{K}(\rho) \rightarrow \infty$, and, therefore, a necessary condition for the partial sums to converge in distribution to a normal limit.

Theorem 2.2. Let $Y_{K}=\sum_{n=0}^{K} \rho^{n} X_{n}$ and $S_{K}(\rho)=\sum_{n=0}^{K} \rho^{2 n}$, where $K \rightarrow \infty$ as $\rho \nearrow 1$. Then

$$
\sup _{x \in D(\rho, K)}\left|\frac{\mathrm{P}\left(Y_{K}>x\right)}{\Phi\left(-x / \sigma \sqrt{S_{K}(\rho)}\right)+1\left(x \geq S_{K}(\rho)^{1 / 2}\right) \bar{F}(x) \sum_{n=0}^{K} \rho^{\alpha n}}-1\right| \rightarrow 0
$$

as $\rho \nearrow 1$, where $D(\rho, K)=\left\{x: x \leq S_{K}(\rho)^{1 / 2}\left(\log S_{K}(\rho)\right)^{1 / 2} \log \log S_{K}(\rho)\right\}$.

When we consider values of $x$ that belong to the heavy-tail domain, the condition that $K \rightarrow \infty$ becomes unnecessary. Moreover, not only can we obtain asymptotics for the distribution of the partial sum $Y_{K}$, but also for its maximum $\bar{Y}_{K}=\max _{0 \leq m \leq K} Y_{m}$. We can also easily add a nonrandom term to $Y_{m}$, say of the form $\rho^{m+1} z$, which allows us to make the connection with the first-passage time distribution of the original AR(1) process.

Define $\tau(x)=\inf \left\{n \geq 0: Z_{n}>x\right\}$. Then, for any $z \in \mathbb{R}$,

$$
\mathrm{P}_{z}(\tau(x) \leq K)=\mathrm{P}_{z}\left(\max _{0 \leq n \leq K} Z_{n}>x\right)=\mathrm{P}\left(\bar{Y}_{K}>x\right) .
$$


There is a considerable literature on the distribution and the expectation of such passage times in the context of both autoregressive processes and their continuous-time counterpart, OrnsteinUhlenbeck processes. Related papers on the first passage time of the AR(1) process are [17] and [2], where an exponential martingale approach and integral equations are used to derive expressions for the distribution of $\tau(x)$ and its expectation. Although we do not pursue this idea here, it is possible that a truncated version of the exponential martingale considered in those papers can provide approximations for the distribution/expectation of $\tau(x)$ that are good in the region of moderate deviations. The result we give below is readily applicable in the region of large deviations of $Y_{K}$.

Theorem 2.3. Let $Y_{m}=\rho^{m+1} z+\sum_{n=0}^{m} \rho^{n} X_{n}$ and $\bar{Y}_{K}=\max _{0 \leq m \leq K} Y_{m}$, where $z \in \mathbb{R}$ is fixed. Define $S_{K}(\rho)=\sum_{n=0}^{K} \rho^{2 n}$. Then, for any $K \geq 0$,

$$
\sup _{x \in C(\rho, K)}\left|\frac{\mathrm{P}\left(Y_{K}>x\right)}{\bar{F}(x) \sum_{n=0}^{K} \rho^{\alpha n}}-1\right| \rightarrow 0
$$

and

$$
\sup _{x \in C(\rho, K)}\left|\frac{\mathrm{P}\left(\bar{Y}_{K}>x\right)}{\bar{F}(x) \sum_{n=0}^{K} \rho^{\alpha n}}-1\right| \rightarrow 0
$$

as $\rho \nearrow 1$, where $C(\rho, K)=\left\{x: x \geq S_{K}(\rho)^{1 / 2}\left(\log S_{K}(\rho)\right)^{1 / 2} v(\rho)\right\}$ and $v(\rho) \rightarrow \infty$ as $\rho \nearrow 1$.

We end this section with a list of related results in the context of autoregressive processes with heavy tails. The distribution of the maximum term of an autoregressive process with innovations that are either exponential or regularly varying was studied in [13], where the first passage times of such processes were also discussed. The extremes of moving average processes with innovations belonging to the domain of max-attraction of the Gumbel distribution (lighter than regularly varying) were studied in [12] and [22]. The stationarity/transience of autoregressive processes with super-heavy-tailed innovations was studied in [25]. In [16] a negative drift random walk with dependent step sizes given by a two-sided linear process with regularly varying innovations was considered, and, as part of their analysis, close to minimal conditions under which (2.5) holds were derived, including the case in which $\alpha \geq 1$. First- and second-order asymptotics for infinite weighted sums with regularly varying tails were given in [1], and generalizations to more general subexponential distributions were given in [14]. Finally, partial weighted sums and their maxima, where the increments belong to a large family of subexponential distributions, were considered in [7].

The rest of the paper contains the proofs of the results in this section. The main proofs are contained in Section 3, and some of the more technical lemmas are contained in Appendix A.

\section{Proofs}

In this section we give the proofs of Theorems 2.1, 2.2, and 2.3. The proof of Corollary 2.1 is a direct consequence of Theorem 2.1 and Lemma 3.1, given below, so its proof is omitted. The section starts with the proofs of Theorems 2.3 and 2.2, which correspond to the analysis of the large and moderate deviations of the partial sums $Y_{K}=\sum_{n=0}^{K} \rho^{n} X_{n}$.

We start by pointing out that the statement of Theorem 2.3 is a special case of a more general theorem from [5], so instead of giving a full proof we just need to show that the conditions for that theorem are satisfied. 
Proof of Theorem 2.3. Write $Y_{m}=\rho^{m+1} z+\sum_{n=0}^{m} \rho^{n} X_{n}$ and $\bar{Y}_{K}=\max _{0 \leq m \leq K} Y_{m}$, where $z \in \mathbb{R}$ is fixed. Let $Z_{m}=\sum_{n=0}^{m} \rho^{n} X_{n}, \bar{Z}_{K}=\max _{0 \leq m \leq K} Z_{m}$,

$$
V(t)=\frac{1}{K+1} \sum_{n=0}^{K} \bar{F}\left(\rho^{-n} t\right), \quad V_{0}(t)=\bar{F}(t), \quad H(K+1)=\sum_{n=0}^{K} \rho^{\alpha n} .
$$

The proof will follow once we show that the conditions of Theorem 13.2.1 of [5] are satisfied. In order to understand the notation used in [5], we need to introduce some definitions. We say that condition $[\cdot,=]_{\mathrm{UR}}$ holds if the functions $V, V_{0}$, and $H$ defined above satisfy

$$
\lim _{K \rightarrow \infty, t \rightarrow \infty} \frac{K V(t)}{H(K) V_{0}(t)}=1 .
$$

(To obtain this definition, we refer the reader to [5, p. 81] for the definition of $[\cdot,=]$ and to $[5$, p. 492] for the definition of [UR]). Note that, by Lemma A.1 in Appendix A,

$$
\lim _{t \rightarrow \infty} \sup _{0<\rho<1} \sup _{K \geq 0}\left|\frac{(K+1) V(t)}{H(K+1) V_{0}(t)}-1\right|=\lim _{t \rightarrow \infty} \sup _{0<\rho<1} \sup _{K \geq 0}\left|\frac{\sum_{n=0}^{K} \bar{F}\left(\rho^{-n} t\right)}{\bar{F}(t) \sum_{n=0}^{K} \rho^{\alpha n}}-1\right|=0,
$$

so condition $[\cdot,=]_{\mathrm{UR}}$ is satisfied. Also,

$$
D_{K+1}:=\sum_{n=0}^{K} \mathrm{E}\left[\left(\rho^{n} X_{n}\right)^{2}\right]=\sigma^{2} \sum_{n=0}^{K} \rho^{2 n}=\sigma^{2} S_{K}(\rho)
$$

so, by Theorem 13.2.1 of [5],

$$
\sup _{x \in C(\rho, K)}\left|\frac{\mathrm{P}\left(\bar{Z}_{K}>x\right)}{\sum_{n=0}^{K} \bar{F}\left(\rho^{-n} x\right)}-1\right| \rightarrow 0, \quad \sup _{x \in C(\rho, K)}\left|\frac{\mathrm{P}\left(Z_{K}>x\right)}{\sum_{n=0}^{K} \bar{F}\left(\rho^{-n} x\right)}-1\right| \rightarrow 0,
$$

as $\rho \nearrow 1$, where $C(\rho, K)=\left\{x: x \geq S_{K}(\rho)^{1 / 2}\left(\log S_{K}(\rho)\right)^{1 / 2} v(\rho)\right\}$ for any $v(\rho) \rightarrow \infty$ as $\rho \nearrow 1$. By Lemma A.1 again we can substitute $\sum_{n=0}^{K} \bar{F}\left(\rho^{-n} x\right)$ by $\bar{F}(x) \sum_{n=0}^{K} \rho^{\alpha n}$ in (3.1). To incorporate the term $\rho^{m+1} z$ into the result, just note that

$$
\mathrm{P}\left(\bar{Y}_{K}>x\right) \leq \mathrm{P}\left(|z|+\bar{Z}_{K}>x\right)=\bar{F}(x-|z|) \sum_{n=0}^{K} \rho^{\alpha n}(1+o(1))
$$

and

$$
\mathrm{P}\left(Y_{K}>x\right) \geq \mathrm{P}\left(-|z|+Z_{K}>x\right)=\bar{F}(x+|z|) \sum_{n=0}^{K} \rho^{\alpha n}(1+o(1)) .
$$

Since $z$ is fixed and $x \rightarrow \infty$ for $x \in C(\rho, K)$, the statement of the theorem follows.

The main idea behind the proof of Theorem 2.2 is to split the probability $\mathrm{P}\left(Y_{K}>x\right)$ into two pieces, i.e.

$$
\mathrm{P}\left(Y_{K}>x\right)=\mathrm{P}\left(Y_{K}>x, \max _{0 \leq n \leq K} \rho^{n} X_{n} \leq y\right)+\mathrm{P}\left(Y_{K}>x, \max _{0 \leq n \leq K} \rho^{n} X_{n}>y\right)
$$

for some carefully chosen $y$. Since the innovations $\left\{X_{i}\right\}$ are heavy tailed, the second term will give rise to the heavy-tailed limit (2.4), while the first term corresponds to the normal limit 
predicted by the Ornstein-Uhlenbeck approximation (2.2). The main technical difficulty lies in the analysis of the first term, for which an exponential change of measure, giving rise to complex asymptotics, is needed. The more technical parts of this analysis are contained in Appendix A.

Before proceeding to the proof of Theorem 2.2 we give two results describing how the sum of the truncated innovations is approximated by the normal distribution, and a third result that will simplify the analysis of (3.2). Proposition 3.1, below, gives the behavior of the first term in (3.2) for tail values larger than the natural range of the normal approximation, that is, larger than $O\left(S_{K}(\rho)^{1 / 2}\right)$. The idea of the proof is to perform an exponential change of measure (since the truncated innovations have finite moment generating function), and to analyze the expression obtained. Proposition 3.2, below, gives this same approximation for tail values of order $S_{K}(\rho)^{1 / 2}$. Finally, Proposition 3.3, below, provides an expression for $\mathrm{P}\left(Y_{K}>x\right)$ that will greatly simplify the proof of Theorem 2.2.

Proposition 3.1. Let $Y_{K, n}=Y_{K}-\rho^{n} X_{n}, S_{K}(\rho)=\sum_{n=0}^{K} \rho^{2 n}$, and $y=y(\rho, K)=S_{K}(\rho)^{1 / 2}$, where $K \rightarrow \infty$ as $\rho \nearrow 1$. Also, define

$$
B(\rho, K)=\left\{x: S_{K}(\rho)^{1 / 2} \leq x \leq S_{K}(\rho)^{1 / 2}\left(\log S_{K}(\rho)\right)^{1 / 2} \log \log S_{K}(\rho)\right\} .
$$

Then, for all $0 \leq n \leq K$ and any $0<\gamma<(\alpha-2) \wedge 1$,

$$
\mathrm{P}\left(Y_{K, n}>u, \max _{0 \leq j \leq K, j \neq n} \rho^{j} X_{j} \leq y\right)=\left(1-\Phi\left(\frac{u}{\sigma \sqrt{S_{K}(\rho)}}\right)\right)\left(1+o\left(y^{-\gamma}\right)\right)
$$

as $\rho \nearrow 1$ and uniformly for $u \in B(\rho, K)$.

Proof. Let $I(K, n)=\{0 \leq i \leq K: i \neq n\}, W_{i}^{(y)}=\rho^{i} X_{i} \mid \rho^{i} X_{i} \leq y$, and $Y_{K, n}^{(y)}=$ $\sum_{i \in I(K, n)} W_{i}^{(y)}$. Then

$$
\mathrm{P}\left(Y_{K, n}>u, \max _{i \in I(K, n)} \rho^{i} X_{i} \leq y\right)=\mathrm{P}\left(Y_{K, n}^{(y)}>u\right) \prod_{i \in I(K, n)} F\left(\rho^{-i} y\right) .
$$

Define $\Lambda_{i, y}(\theta)=\log \mathrm{E}\left[\mathrm{e}^{\theta W_{i}^{(y)}}\right], F_{i, y}(x)=\mathrm{P}\left(W_{i}^{(y)} \leq x\right)=F\left(\rho^{-i}(x \wedge y)\right) / F\left(\rho^{-i} y\right)$, and $F_{\theta, i, y}(\mathrm{~d} x)=\mathrm{e}^{\theta x-\Lambda_{i, y}(\theta)} F_{i, y}(\mathrm{~d} x)$. Note that

$$
\begin{aligned}
\mathrm{P}\left(Y_{K, n}^{(y)}>u\right) & \\
& =\int \cdots \int_{R} F_{0, y}\left(\mathrm{~d} w_{0}\right) \cdots F_{K, y}\left(\mathrm{~d} w_{K}\right) \\
& =\exp \left(\sum_{i \in(K, n)} \Lambda_{i, y}(\theta)\right) \int \cdots \int_{R} \exp \left(-\sum_{i \in I(K, n)} \theta w_{i}\right) F_{\theta, 0, y}\left(\mathrm{~d} w_{0}\right) \cdots F_{\theta, K, y}\left(\mathrm{~d} w_{K}\right) \\
& =\exp \left(-\theta u+\sum_{i \in I(K, n)} \Lambda_{i, y}(\theta)\right) \mathrm{E}\left[\mathrm{e}^{-\theta\left(\tilde{Y}_{K, n}^{(y)}-u\right)} 1\left(\tilde{Y}_{K, n}^{(y)}>u\right)\right],
\end{aligned}
$$

where $R=\left\{\left(w_{0}, \ldots, w_{K}\right): \sum_{i \in I(K, n)} w_{i}>u\right\}, \tilde{Y}_{K, n}^{(y)}=\sum_{i \in I(K, n)} V_{i}$, and $\left\{V_{n}: n \geq 0\right\}$ are independent random variables with $\mathrm{P}\left(V_{i} \leq z\right)=F_{\theta, i, y}(z)$. Define $\hat{\theta}=\hat{\theta}(\rho, K, u, n)$ to be the solution of the equation

$$
-u+\sum_{i \in I(K, n)} \Lambda_{i, y}^{\prime}(\hat{\theta})=0 .
$$


Choose $2+\gamma<\beta<\alpha \wedge 3$. By Lemma A.3,

$$
\hat{\theta}=\frac{u}{\sigma^{2} S_{K}(\rho)}+o\left(\left(\frac{u}{S_{K}(\rho)}\right)^{\beta-1}\right)=\frac{u}{\sigma^{2} S_{K}(\rho)}+o\left(u^{-1} y^{-\gamma}\right)
$$

uniformly for $u \in B(\rho, K)$. By Lemma A.4,

$$
\exp \left(\sum_{i \in I(K, n)} \Lambda_{i, y}(\hat{\theta})\right) \prod_{i \in I(K, n)} F\left(\rho^{-i} y\right)=\exp \left(\frac{u^{2}}{2 \sigma^{2} S_{K}(\rho)}+o\left(y^{-(\beta-2)}\right)\right) .
$$

Combining these two expressions we obtain

$$
\begin{aligned}
& \mathrm{P}\left(Y_{K, n}>u, \max _{0 \leq j \leq K, j \neq n} \rho^{j} X_{j} \leq y\right) \\
& \quad=\exp \left(-\frac{u^{2}}{2 \sigma^{2} S_{K}(\rho)}+o\left(y^{-\gamma}\right)\right) \mathrm{E}\left[\mathrm{e}^{-\hat{\theta}\left(\tilde{Y}_{K, n}^{(y)}-u\right)} 1\left(\tilde{Y}_{K, n}^{(y)}>u\right)\right] .
\end{aligned}
$$

To analyze the remaining expectation, note that

$$
\mathrm{E}\left[V_{i}\right]=\mathrm{E}\left[W_{i}^{(y)} \mathrm{e}^{\hat{\theta} W_{i}^{(y)}}\right] \frac{1}{\mathrm{E}\left[\mathrm{e}^{\hat{\theta} W_{i}^{(y)}}\right]}=\Lambda_{i, y}^{\prime}(\hat{\theta})
$$

so our choice of $\hat{\theta}$ gives

$$
\mathrm{E}\left[\tilde{Y}_{K, n}^{(y)}\right]=\sum_{i \in I(K, n)} \Lambda_{i, y}^{\prime}(\hat{\theta})=u .
$$

Define $\hat{V}_{i}=V_{i}-\mathrm{E}\left[V_{i}\right], B_{K}=\sum_{i \in I(K, n)} \mathrm{E}\left[\hat{V}_{i}^{2}\right], U_{K}=B_{K}^{-1 / 2} \sum_{i \in I(K, n)} \hat{V}_{i}$, and $G_{K}(u)=$ $\mathrm{P}\left(U_{K} \leq u\right)$. Let $\gamma<\delta<(\alpha-2) \wedge 1$. Then, by a generalization of Esseen's inequality (see [20, Theorem 5.6, p. 151]),

$$
\sup _{u}\left|G_{K}(u)-\Phi(u)\right| \leq \frac{C}{B_{K}^{1+\delta / 2}} \sum_{i \in I(K, n)} \mathrm{E}\left[\left|\hat{V}_{i}\right|^{2+\delta}\right]
$$

for some constant $C>0$. Therefore,

$$
\begin{aligned}
& \mathrm{E}\left[\mathrm{e}^{-\hat{\theta}\left(\tilde{Y}_{K, n}^{(y)}-u\right)} 1\left(\tilde{Y}_{K, n}^{(y)}>u\right)\right] \\
& \quad=\mathrm{E}\left[\mathrm{e}^{-\hat{\theta} B_{K}^{1 / 2} U_{K}} 1\left(U_{K}>0\right)\right] \\
& \quad=\int_{0}^{\infty} \mathrm{e}^{-\hat{\theta} B_{K}^{1 / 2} t} G_{K}(\mathrm{~d} t) \\
& \quad=-G_{K}(0)+\hat{\theta} B_{K}^{1 / 2} \int_{0}^{\infty} \mathrm{e}^{-\hat{\theta} B_{K}^{1 / 2} t} G_{K}(t) \mathrm{d} t \\
& \quad=-\Phi(0)+\hat{\theta} B_{K}^{1 / 2} \int_{0}^{\infty} \mathrm{e}^{-\hat{\theta} B_{K}^{1 / 2} t} \Phi(t) \mathrm{d} t+E(\rho, K, u, n),
\end{aligned}
$$

where $\phi(t)=\mathrm{e}^{-t^{2} / 2} / \sqrt{2 \pi}, \Phi(t)=\int_{-\infty}^{t} \phi(s) \mathrm{d} s$, and

$$
E(\rho, K, u, n)=\left(\Phi(0)-G_{K}(0)\right)+\int_{0}^{\infty} \mathrm{e}^{-s}\left(G_{K}\left(\frac{s}{\hat{\theta} B_{K}^{1 / 2}}\right)-\Phi\left(\frac{s}{\hat{\theta} B_{K}^{1 / 2}}\right)\right) \mathrm{d} s .
$$


By Lemma A.4,

$$
\mathrm{E}\left[\hat{V}_{i}^{2}\right]=\rho^{2 i} \sigma^{2}+o\left(\rho^{i \beta} y^{-(\beta-2)}\right), \quad \mathrm{E}\left[\left|\hat{V}_{i}\right|^{2+\delta}\right]=O\left(\mathrm{e}^{\hat{\theta} y} \rho^{(2+\delta) i}\right),
$$

which gives

$$
B_{K}=\sum_{i \in I(K, n)}\left(\rho^{2 i} \sigma^{2}+o\left(\rho^{i \beta} y^{-(\beta-2)}\right)\right)=\sigma^{2} S_{K}(\rho)-\sigma^{2} \rho^{2 n}+o\left(S_{K}(\rho) y^{-(\beta-2)}\right) .
$$

Combining (3.5) with (3.4) yields

$$
\begin{aligned}
|E(\rho, K, u, n)| & \leq \frac{C}{B_{K}^{1+\delta / 2}} \sum_{i \in I(K, n)} \mathrm{E}\left[\left|\hat{V}_{i}\right|^{2+\delta}\right] \\
& =O\left(\frac{\mathrm{e}^{\hat{\theta} y}}{S_{K}(\rho)^{1+\delta / 2}} \sum_{i=0}^{K} \rho^{(2+\delta) i}\right) \\
& =O\left(\mathrm{e}^{\hat{\theta} y} y^{-\delta}\right) .
\end{aligned}
$$

Finally, straightforward computation gives

$$
\begin{aligned}
-\Phi(0)+\hat{\theta} B_{K}^{1 / 2} \int_{0}^{\infty} \mathrm{e}^{-\hat{\theta} B_{K}^{1 / 2} t} \Phi(t) \mathrm{d} t & =\int_{0}^{\infty} \mathrm{e}^{-\hat{\theta} B_{K}^{1 / 2} t} \phi(t) \mathrm{d} t \\
& =\mathrm{e}^{\hat{\theta}^{2} B_{K} / 2} \int_{0}^{\infty} \frac{1}{\sqrt{2 \pi}} \mathrm{e}^{-\left(t+\hat{\theta} B_{K}^{1 / 2}\right)^{2} / 2} \mathrm{~d} t \\
& =\mathrm{e}^{\hat{\theta}^{2} B_{K} / 2} \int_{\hat{\theta} B_{K}^{1 / 2}}^{\infty} \frac{1}{\sqrt{2 \pi}} \mathrm{e}^{-w^{2} / 2} \mathrm{~d} w \\
& =\mathrm{e}^{\hat{\theta}^{2} B_{K} / 2} \Phi\left(-\hat{\theta} B_{K}^{1 / 2}\right) .
\end{aligned}
$$

Combining (3.3) and (3.5) gives

$$
\begin{aligned}
\hat{\theta} B_{K}^{1 / 2} & =\left(\frac{u}{\sigma^{2} S_{K}(\rho)}\right) \sigma S_{K}(\rho)^{1 / 2}\left(1+o\left(\left(\frac{u}{S_{K}(\rho)}\right)^{\beta-2}\right)\right)\left(1+O\left(S_{K}(\rho)^{-1}\right)\right) \\
& =\frac{u}{\sigma S_{K}(\rho)^{1 / 2}}+o\left(\frac{u^{\beta-1}}{S_{K}(\rho)^{\beta-3 / 2}}\right),
\end{aligned}
$$

from which we also obtain

$$
\frac{\hat{\theta}^{2} B_{K}}{2}=\frac{u^{2}}{2 \sigma^{2} S_{K}(\rho)}+o\left(\frac{u^{\beta}}{S_{K}(\rho)^{\beta-1}}\right)=\frac{u^{2}}{2 \sigma^{2} S_{K}(\rho)}+o\left(y^{-\gamma}\right) .
$$

Let $z=u / \sqrt{\sigma^{2} S_{K}(\rho)}$. Then

$$
\begin{aligned}
\mathrm{E}\left[\mathrm{e}^{-\hat{\theta}\left(\tilde{Y}_{K, n}^{(y)}-u\right)} 1\left(\tilde{Y}_{K, n}^{(y)}>u\right)\right] \\
\quad=\mathrm{e}^{\hat{\theta}^{2} B_{K} / 2} \Phi\left(-\hat{\theta} B_{K}^{1 / 2}\right)+O\left(\mathrm{e}^{\hat{\theta} y} y^{-\delta}\right) \\
\quad=\mathrm{e}^{z^{2} / 2+o\left(y^{-\gamma}\right)} \Phi\left(-z+o\left(z^{\beta-1} y^{2-\beta}\right)\right)+O\left(\mathrm{e}^{\hat{\theta} y} y^{-\delta}\right) \\
\quad=\mathrm{e}^{z^{2} / 2+o\left(y^{-\gamma}\right)}\left(\Phi(-z)+o\left(z^{\beta-1} y^{2-\beta} \phi(z)\right)\right)+O\left(\mathrm{e}^{\hat{\theta} y} y^{-\delta}\right) \\
\quad=\mathrm{e}^{z^{2} / 2+o\left(y^{-\gamma}\right)} \Phi(-z)\left(1+o\left(\frac{\phi(z) z^{\beta} y^{2-\beta}}{z \Phi(-z)}\right)+O\left(\frac{\phi(z) \mathrm{e}^{\hat{\theta} y} z y^{-\delta}}{z \Phi(-z)}\right)\right) .
\end{aligned}
$$


Since, for $u \in B(\rho, K)$, we have $c_{1} \leq z \leq c_{2}(\log y)^{1 / 2} \log \log y$ for some constants $c_{1}, c_{2}>0$ and $z \Phi(-z) \sim \phi(z)$ as $z \rightarrow \infty$, then

$$
\mathrm{E}\left[\mathrm{e}^{-\hat{\theta}\left(\tilde{Y}_{K, n}^{(y)}-u\right)} 1\left(\tilde{Y}_{K, n}^{(y)}>u\right)\right]=\mathrm{e}^{z^{2} / 2+o\left(y^{-\gamma}\right)} \Phi(-z)\left(1+o\left(z^{\beta} y^{2-\beta}\right)+O\left(\mathrm{e}^{\hat{\theta} y} z y^{-\delta}\right)\right)
$$

Also, since $y \hat{\theta}=O\left((\log y)^{1 / 2} \log \log y\right)$, then both $z^{\beta}$ and $\mathrm{e}^{\hat{\theta} y} z$ are bounded by slowly varying functions of $y$, and the facts that $\beta-2>\gamma$ and $\delta>\gamma$ give

$$
\mathrm{E}\left[\mathrm{e}^{-\hat{\theta}\left(\tilde{Y}_{K, n}^{(y)}-u\right)} 1\left(\tilde{Y}_{K, n}^{(y)}>u\right)\right]=\mathrm{e}^{z^{2} / 2+o\left(y^{-\gamma}\right)} \Phi(-z)\left(1+o\left(y^{-\gamma}\right)\right) .
$$

We conclude that

$$
\mathrm{P}\left(Y_{K, n}>u, \max _{0 \leq j \leq K, j \neq n} \rho^{j} X_{j} \leq y\right)=(1-\Phi(z))\left(1+o\left(y^{-\gamma}\right)\right),
$$

which completes the proof.

Proposition 3.2. Let $Y_{K, n}=Y_{K}-\rho^{n} X_{n}, S_{K}(\rho)=\sum_{n=0}^{K} \rho^{2 n}$, and $y=y(\rho, K)=S_{K}(\rho)^{1 / 2}$, where $K \rightarrow \infty$ as $\rho \nearrow 1$. Also, define

$$
A(\rho, K)=\left\{x: x \leq S_{K}(\rho)^{1 / 2}\right\} .
$$

Then, for all $0 \leq n \leq K$ and any $0<\gamma<(\alpha-2) \wedge 1$,

$$
\mathrm{P}\left(Y_{K, n}>u, \max _{0 \leq j \leq K, j \neq n} \rho^{j} X_{j} \leq y\right)=\left(1-\Phi\left(\frac{u}{\sigma \sqrt{S_{K}(\rho)}}\right)\right)\left(1+o\left(y^{-\gamma}\right)\right)
$$

as $\rho \nearrow 1$, uniformly for $u \in A(\rho, K)$.

Proof. We start by writing

$$
\begin{aligned}
& \mathrm{P}\left(Y_{K, n}>u, \max _{0 \leq j \leq K, j \neq n} \rho^{j} X_{j} \leq y\right) \\
& =\mathrm{P}\left(Y_{K, n}>u\right)-\mathrm{P}\left(Y_{K, n}>u, \max _{0 \leq j \leq K, j \neq n} \rho^{j} X_{j}>y\right) \\
& =\mathrm{P}\left(Y_{K, n}>u\right)+O\left(\mathrm{P}\left(\max _{0 \leq j \leq K, j \neq n} \rho^{j} X_{j}>y\right)\right) .
\end{aligned}
$$

To analyze the second term, note that

$$
\begin{aligned}
\mathrm{P}\left(\max _{0 \leq j \leq K, j \neq n} \rho^{j} X_{j}>y\right) & \leq \mathrm{P}\left(\bigcup_{i=0}^{K}\left\{\rho^{i} X_{i}>y\right\}\right) \\
& \leq \sum_{i=0}^{K} \bar{F}\left(\rho^{-i} y\right) \\
& =O\left(\bar{F}(y) \sum_{j=0}^{K} \rho^{\alpha j}\right) \\
& =O\left(y^{2} \bar{F}(y)\right),
\end{aligned}
$$

where the second equality is justified by Lemma A.1. Now, to analyze $\mathrm{P}\left(Y_{K, n}>u\right)$, we will 
use a generalization of the Berry-Esseen theorem. Define $Q_{i}=\rho^{i} X_{i}$ and

$$
B_{K}=\sum_{0 \leq j \leq K, j \neq n} \operatorname{var}\left(Q_{j}\right)=\sum_{0 \leq i \leq K, i \neq n} \rho^{2 i} \sigma^{2} .
$$

Fix $\gamma<\delta<(\alpha-2) \wedge 1$. Then $\mathrm{E}\left[\left|Q_{j}\right|^{2+\delta}\right]=\rho^{(2+\delta) j} \mathrm{E}\left[\left|X_{j}\right|^{2+\delta}\right]<\infty$ for all $j$. Then, by Theorem 5.6 of [20, p. 151],

$$
\sup _{u}\left|\mathrm{P}\left(Y_{K, n}>u\right)-\Phi\left(-u B_{K}^{-1 / 2}\right)\right| \leq \frac{C}{B_{K}^{1+\delta / 2}} \sum_{j=0}^{K} \mathrm{E}\left[\left|Q_{j}\right|^{2+\delta}\right]
$$

for some constant $C>0$, where $\Phi$ is the standard normal distribution function. To obtain a bound for the error, note that

$$
\begin{aligned}
\frac{1}{B_{K}^{1+\delta / 2}} \sum_{j=0}^{K} \mathrm{E}\left[\left|Q_{j}\right|^{2+\delta}\right] & =\frac{\mathrm{E}\left[\left|X_{1}\right|^{2+\delta}\right]}{\left[\sigma^{2}\left(S_{K}(\rho)-\rho^{2 n}\right)\right]^{1+\delta / 2}} \sum_{j=0}^{K} \rho^{(2+\delta) j} \\
& =O\left(S_{K}(\rho)^{-1-\delta / 2} \sum_{j=0}^{K} \rho^{(2+\delta) j}\right) \\
& =O\left(y^{-\delta}\right) .
\end{aligned}
$$

It follows that

$$
\mathrm{P}\left(Y_{K, n}>u\right)=\Phi\left(-u B_{K}^{-1 / 2}\right)+O\left(y^{-\delta}\right),
$$

which combined with (3.6) gives

$$
\mathrm{P}\left(Y_{K, n}>u, \max _{0 \leq j \leq K, j \neq n} \rho^{j} X_{j} \leq y\right)=\Phi\left(-u B_{K}^{-1 / 2}\right)+O\left(y^{-\delta}+y^{2} \bar{F}(y)\right) .
$$

Since $B_{K}=\sigma^{2}\left(S_{K}(\rho)-\rho^{2 n}\right)=\sigma^{2} y^{2}\left(1-\rho^{2 n} y^{-2}\right)$, then, by using the inequality

$$
1 \leq(1-t)^{-1 / 2} \leq 1+\frac{(1-t)^{-3 / 2} t}{2} \text { for } t>0,
$$

we obtain

$$
\begin{aligned}
\Phi\left(-\frac{u}{\sigma y}\right) & \geq \Phi\left(-u B_{K}^{-1 / 2}\right) \\
& \geq \Phi\left(-\frac{u}{\sigma y}\left(1+\frac{\rho^{2 n}}{2 y^{2}\left(1-\rho^{2 n} y^{-2}\right)^{3 / 2}}\right)\right) \\
& \geq \Phi\left(-\frac{u}{\sigma y}\right)-\phi\left(\frac{|u|}{\sigma y}\right) \frac{|u| \rho^{2 n}}{2 \sigma y^{3}\left(1-\rho^{2 n} y^{-2}\right)^{3 / 2}} \\
& =\Phi\left(-\frac{u}{\sigma y}\right)\left(1+O\left(\frac{\phi(|u| /(\sigma y))|u|}{y^{3} \Phi(-u /(\sigma y))}\right)\right) .
\end{aligned}
$$

Since, for $u \leq y$, we have

$$
\Phi\left(-\frac{u}{\sigma y}\right) \geq \Phi\left(-\frac{1}{\sigma}\right)>0 \quad \text { and } \quad \phi\left(\frac{|u|}{\sigma y}\right)|u| y^{-3}=o\left(y^{-2}\right)
$$


it follows that

$$
\Phi\left(-u B_{K}^{-1 / 2}\right)=\Phi\left(-\frac{u}{\sigma y}\right)\left(1+o\left(y^{-2}\right)\right) .
$$

Finally, noting that $y^{-\delta}=o\left(y^{-\gamma}\right)$ and $y^{2} \bar{F}(y)=o\left(y^{-\gamma}\right)$ gives

$$
\begin{aligned}
\mathrm{P}\left(Y_{K, n}>u, \max _{0 \leq j \leq K, j \neq n} \rho^{j} X_{j} \leq y\right) & =\Phi\left(-\frac{u}{\sigma y}\right)\left(1+o\left(y^{-2}\right)\right)+o\left(y^{-\gamma}\right) \\
& =\Phi\left(-\frac{u}{\sigma y}\right)\left(1+o\left(y^{-\gamma}\right)\right) .
\end{aligned}
$$

We now use the two previous results to obtain an expression for $\mathrm{P}\left(Y_{K}>x\right)$ suitable for the proof of Theorem 2.2.

Proposition 3.3. Let $S_{K}(\rho)=\sum_{n=0}^{K} \rho^{2 n}, y=y(\rho, K)=S_{K}(\rho)^{1 / 2}$, and

$$
D(\rho, K)=\left\{x: x \leq S_{K}(\rho)^{1 / 2}\left(\log S_{K}(\rho)\right)^{1 / 2} \log \log S_{K}(\rho)\right\}
$$

where $K \rightarrow \infty$ as $\rho \nearrow 1$. Then, for any $0<\gamma<(\alpha-2) \wedge 1$,

$$
\mathrm{P}\left(Y_{K}>x\right)=\left(\Phi\left(-\frac{x}{\sigma y}\right)+\sum_{n=0}^{K} \int_{y}^{\infty} \Phi\left(-\frac{(x-t)}{\sigma y}\right) \mathrm{P}\left(\rho^{n} X_{n} \in \mathrm{d} t\right)\right)\left(1+o\left(y^{-\gamma}\right)\right)
$$

as $\rho \nearrow 1$, uniformly for $x \in D(\rho, K)$.

Proof. Define

$$
N_{y}=\#\left\{0 \leq j \leq K: \rho^{j} X_{j}>y\right\} .
$$

We start by noting that $Y_{K}=Y_{K+1, K+1}$ in Propositions 3.1 and 3.2, so by these results we obtain

$$
\mathrm{P}\left(Y_{K}>x, N_{y}=0\right)=\Phi\left(-\frac{x}{\sigma y}\right)\left(1+o\left(y^{-\gamma}\right)\right) .
$$

We can bound $\mathrm{P}\left(Y_{K}>x, N_{y} \geq 2\right)$ as follows:

$$
\begin{aligned}
\mathrm{P}\left(Y_{K}>x, N_{y} \geq 2\right) & \leq \mathrm{P}\left(N_{y} \geq 2\right) \\
& =\mathrm{P}\left(\bigcup_{0 \leq i \neq j \leq K}\left\{\rho^{i} X_{i}>y\right\} \cap\left\{\rho^{j} X_{j}>y\right\}\right) \\
& \leq \sum_{0 \leq i \neq j \leq K} \bar{F}\left(\rho^{-i} y\right) \bar{F}\left(\rho^{-j} y\right) \\
& \leq\left(\sum_{i=0}^{K} \bar{F}\left(\rho^{-i} y\right)\right)^{2} \\
& =O\left(\bar{F}(y)^{2}\left(\sum_{n=0}^{K} \rho^{\alpha n}\right)^{2}\right) \\
& =O\left(y^{4} \bar{F}(y)^{2}\right)
\end{aligned}
$$


where the last equality is justified by Lemma A.1. We now proceed to bound $\mathrm{P}\left(Y_{K}>x\right.$, $\left.N_{y}=1\right)$. Define $Y_{K, n}=Y_{K}-\rho^{n} X_{n}$, and note that

$$
\begin{aligned}
\mathrm{P}\left(Y_{K}\right. & \left.>x, N_{y}=1\right) \\
& =\sum_{n=0}^{K} \mathrm{P}\left(Y_{K}>x, \rho^{n} X_{n}>y, \max _{0 \leq j \leq K, j \neq n} \rho^{j} X_{j} \leq y\right) \\
& =\sum_{n=0}^{K} \int_{y}^{\infty} \mathrm{P}\left(Y_{K, n}>x-t, \max _{0 \leq j \leq K, j \neq n} \rho^{j} X_{j} \leq y\right) \mathrm{P}\left(\rho^{n} X_{n} \in \mathrm{d} t\right) \\
& =\sum_{n=0}^{K} \int_{y}^{\infty} \Phi\left(-\frac{(x-t)}{\sigma y}\right) \mathrm{P}\left(\rho^{n} X_{n} \in \mathrm{d} t\right)\left(1+o\left(y^{-\gamma}\right)\right),
\end{aligned}
$$

where the last step is justified by Propositions 3.1 and 3.2. Combining the estimates for $\mathrm{P}\left(Y_{K}>x, N_{y}=0\right), \mathrm{P}\left(Y_{K}>x, N_{y}=1\right)$, and $\mathrm{P}\left(Y_{K}>x, N_{y} \geq 2\right)$, we obtain

$$
\begin{aligned}
\mathrm{P}\left(Y_{K}>x\right)= & \left(\Phi\left(-\frac{x}{\sigma y}\right)+\sum_{n=0}^{K} \int_{y}^{\infty} \Phi\left(-\frac{(x-t)}{\sigma y}\right) \mathrm{P}\left(\rho^{n} X_{n} \in \mathrm{d} t\right)\right)\left(1+o\left(y^{-\gamma}\right)\right) \\
& +O\left(y^{4} \bar{F}(y)^{2}\right) .
\end{aligned}
$$

Finally, note that, for $x \in D(\rho, K)$, we have $x \leq y\left(\log y^{2}\right)^{1 / 2}\left(\log \log y^{2}\right)$. Define $\hat{L}(y)=$ $\left(\log y^{2}\right)^{1 / 2}\left(\log \log y^{2}\right)$. Then

$$
\begin{aligned}
& \Phi\left(-\frac{x}{\sigma y}\right)+\sum_{n=0}^{K} \int_{y}^{\infty} \Phi\left(-\frac{(x-t)}{\sigma y}\right) \mathrm{P}\left(\rho^{n} X_{n} \in \mathrm{d} t\right) \\
& \quad \geq \sum_{n=0}^{K} \int_{y}^{\infty} \Phi\left(-\frac{(y \hat{L}(y)-t)}{\sigma y}\right) \mathrm{P}\left(\rho^{n} X_{n} \in \mathrm{d} t\right) \\
& \quad=\sum_{n=0}^{K}\left(\Phi\left(-\frac{(\hat{L}(y)-1)}{\sigma}\right) \bar{F}\left(\rho^{-n} y\right)+\int_{(1-\hat{L}(y)) / \sigma}^{\infty} \phi(u) \bar{F}\left(\rho^{-n}(y \hat{L}(y)+\sigma y u)\right) \mathrm{d} u\right) \\
& \geq \sum_{n=0}^{K} \bar{F}\left(\rho^{-n} y \hat{L}(y)\right) \int_{(1-\hat{L}(y)) / \sigma}^{0} \phi(u) \mathrm{d} u \\
& =\left(\Phi\left(\frac{\hat{L}(y)-1}{\sigma}\right)-\frac{1}{2}\right) \sum_{n=0}^{K} \bar{F}\left(\rho^{-n} y \hat{L}(y)\right) .
\end{aligned}
$$

Since $y \hat{L}(y) \rightarrow \infty$ and $\hat{L}(y) \rightarrow \infty$, then, by Lemma A.1,

$$
\frac{y^{4} \bar{F}(y)^{2}}{\Phi(-x /(\sigma y))+\sum_{n=0}^{K} \int_{y}^{\infty} \Phi(-(x-t) /(\sigma y)) \mathrm{P}\left(\rho^{n} X_{n} \in \mathrm{d} t\right)}=O\left(\frac{y^{4} \bar{F}(y)^{2}}{\bar{F}(y \hat{L}(y)) \sum_{n=0}^{K} \rho^{\alpha n}}\right) .
$$

Note that

$$
\frac{y^{2}}{\sum_{n=0}^{K} \rho^{\alpha n}}=\frac{\left(1-\rho^{2(K+1)}\right)\left(1-\rho^{\alpha}\right)}{\left(1-\rho^{\alpha(K+1)}\right)\left(1-\rho^{2}\right)} \leq \frac{1-\rho^{\alpha}}{1-\rho^{2}} \rightarrow \frac{\alpha}{2} \quad \text { as } \rho \nearrow 1 .
$$


It follows that

$$
\frac{y^{4} \bar{F}(y)^{2}}{\bar{F}(y \hat{L}(y)) \sum_{n=0}^{K} \rho^{\alpha n}}=O\left(\frac{y^{2} \bar{F}(y)^{2}}{\bar{F}(y \hat{L}(y))}\right)=O\left(\frac{y^{-(\alpha-2)} L(y)^{2} \hat{L}(y)^{\alpha}}{L(y \hat{L}(y))}\right)=o\left(y^{-\gamma}\right) .
$$

This completes the proof.

We are now ready to give the proof of Theorem 2.2, which includes the asymptotics of $\mathrm{P}\left(Y_{K}>x\right)$ for both the region where the normal approximation is valid and the region where the transition from the normal approximation into the heavy-tailed asymptotic occurs.

Proof of Theorem 2.2. Fix $0<\gamma<(\alpha-2) \wedge 1$, and let $y=S_{K}(\rho)^{1 / 2}$. Then, by Proposition 3.3,

$$
\mathrm{P}\left(Y_{K}>x\right)=\left(\Phi\left(-\frac{x}{\sigma y}\right)+\sum_{n=0}^{K} \int_{y}^{\infty} \Phi\left(-\frac{(x-t)}{\sigma y}\right) \mathrm{P}\left(\rho^{n} X_{n} \in \mathrm{d} t\right)\right)\left(1+o\left(y^{-\gamma}\right)\right)
$$

as $\rho \nearrow 1$, uniformly for $x \in D(\rho, K)$. Let

$$
\begin{gathered}
Z(x, \rho, K)=\Phi\left(-\frac{x}{\sigma y}\right)+1(x \geq y) \bar{F}(x) \sum_{n=0}^{K} \rho^{\alpha n}, \\
I(x, \rho, K)=\sum_{n=0}^{K} \int_{y}^{\infty} \Phi\left(-\frac{(x-t)}{\sigma y}\right) \mathrm{P}\left(\rho^{n} X_{n} \in \mathrm{d} t\right), \\
\text { and } J(x, y)=\int_{y}^{\infty} \Phi\left(-\frac{(x-t)}{\sigma y}\right) F(\mathrm{~d} t) .
\end{gathered}
$$

Then, it only remains to prove that

$$
\sup _{x \in D(\rho, K)}\left|\frac{I(x, \rho, K)-1(x \geq y) \bar{F}(x) \sum_{n=0}^{K} \rho^{\alpha n}}{Z(x, \rho, K)}\right| \rightarrow 0
$$

as $\rho \nearrow 1$. First let $w=w(y)=\sigma y(c \log y)^{1 / 2}$, with $0<c<2(\alpha-2-\gamma)$, and use the equivalence $\Phi(-x) \sim x^{-1} \phi(x)$ as $x \rightarrow \infty$ to obtain

$$
\begin{aligned}
\sup _{x<w}\left|\frac{I(x, \rho, K)-1(x \geq y) \bar{F}(x) \sum_{n=0}^{K} \rho^{\alpha n}}{Z(x, \rho, K)}\right| & \leq \frac{1}{\Phi(-w /(\sigma y))} \sum_{n=0}^{K}\left(\bar{F}\left(\rho^{-n} y\right)+\bar{F}(y) \rho^{\alpha n}\right) \\
& =O\left(\frac{(\log y)^{1 / 2} \bar{F}(y)}{\phi\left((c \log y)^{1 / 2}\right)} \sum_{n=0}^{K} \rho^{\alpha n}\right) \\
& =O\left((\log y)^{1 / 2} y^{2+c / 2} \bar{F}(y)\right) \\
& =o\left(y^{-\gamma}\right),
\end{aligned}
$$

where in the first equality we used Lemma A.1. To analyze the supremum over the remaining values of $x$, let $G(\rho, K)=\left\{x: w \leq x \leq y\left(\log y^{2}\right)^{1 / 2} \log \log y^{2}\right\}$ (note that, for sufficiently 
large values of $y$, we have $\left.y \leq w \leq y\left(\log y^{2}\right)^{1 / 2} \log \log y^{2}\right)$ and note that

$$
\begin{aligned}
& \sup _{x \in G(\rho, K)}\left|\frac{I(x, \rho, K)-1(x \geq y) \bar{F}(x) \sum_{n=0}^{K} \rho^{\alpha n}}{Z(x, \rho, K)}\right| \\
& \quad \leq \sup _{x \in G(\rho, K)}\left|\frac{J(x, y)-\bar{F}(x)}{\bar{F}(x)}\right|+\sup _{x \in G(\rho, K)}\left|\frac{I(x, \rho, K)-J(x, y) \sum_{n=0}^{K} \rho^{\alpha n}}{\bar{F}(x) \sum_{n=0}^{K} \rho^{\alpha n}}\right| .
\end{aligned}
$$

To bound the first supremum in (3.7), integrate by parts and use a change of variables to obtain

$$
\begin{aligned}
& \sup _{x \in G(\rho, K)}\left|\frac{J(x, y)-\bar{F}(x)}{\bar{F}(x)}\right| \\
& \quad=\sup _{x \in G(\rho, K)}\left|\frac{\Phi(-(x-y) /(\sigma y)) \bar{F}(y)+\int_{-(x-y) /(\sigma y)}^{\infty} \phi(u) \bar{F}(x+\sigma y u) \mathrm{d} u-\bar{F}(x)}{\bar{F}(x)}\right| \\
& =\sup _{x \in G(\rho, K)}\left|\mathrm{E}\left[\frac{\bar{F}(y \vee(x+\sigma y Z))}{\bar{F}(x)}-1\right]\right|,
\end{aligned}
$$

where $Z$ is a standard normal random variable. It follows that

$$
\begin{aligned}
\sup _{x \in G(\rho, K)} & \left|\frac{J(x, y)-\bar{F}(x)}{\bar{F}(x)}\right| \\
\leq & \sup _{x \in G(\rho, K)} \mathrm{E}\left[\left|\frac{\bar{F}(x+\sigma y Z)}{\bar{F}(x)}-1\right| 1\left(|Z| \leq \frac{x}{\sigma y \log \log y}\right)\right] \\
& +\sup _{x \in G(\rho, K)} \mathrm{E}\left[\left|\frac{\bar{F}(y \vee(x+\sigma y Z))}{\bar{F}(x)}-1\right| 1\left(|Z|>\frac{x}{\sigma y \log \log y}\right)\right],
\end{aligned}
$$

where we used the fact that, when $|Z| \leq x /(\sigma y \log \log y)$ and $x \in G(\rho, K)$, we have

$$
x+\sigma y Z \geq x-\sigma y \frac{x}{\sigma y \log \log y} \geq w\left(1-\frac{1}{\log \log y}\right) \gg y
$$

for sufficiently large values of $y$. Note that (3.8) is bounded by

$$
\sup _{x \in G(\rho, K)} \max \left\{\left|\frac{\bar{F}(x+x / \log \log y)}{\bar{F}(x)}-1\right|,\left|\frac{\bar{F}(x-x / \log \log y)}{\bar{F}(x)}-1\right|\right\},
$$

which converges to 0 by basic properties of regularly varying functions. The expectation in (3.9) is bounded by

$$
\sup _{x \in G(\rho, K)}\left(\frac{\bar{F}(y)}{\bar{F}(x)}+1\right) 2 \Phi\left(-\frac{x}{\sigma y \log \log y}\right),
$$

which, by Potter's theorem (see Theorem 1.5.6(ii) of [4, p. 25]), is in turn bounded by

$$
\begin{aligned}
\sup _{x \in G(\rho, K)} A\left(\frac{x}{y}\right)^{\alpha+\delta} \Phi\left(-\frac{x}{\sigma y \log \log y}\right) & =A\left(\frac{w}{y}\right)^{\alpha+\delta} \Phi\left(-\frac{w}{\sigma y \log \log y}\right) \\
& =A^{\prime}(\log y)^{(\alpha+\delta) / 2} \Phi\left(-\frac{(c \log y)^{1 / 2}}{\log \log y}\right)
\end{aligned}
$$


for some constants $A, A^{\prime}>1$ and $\delta>0$. Since the above converges to 0 as $y \rightarrow \infty$, we have shown that

$$
\sup _{x \in G(\rho, K)}\left|\frac{J(x, y)-\bar{F}(x)}{\bar{F}(x)}\right| \rightarrow 0 .
$$

To analyze the second supremum in (3.7), integrate by parts to obtain

$$
\begin{aligned}
\sup _{x \in G(\rho, K)}\left|\frac{I(x, \rho, K)-J(x, y) \sum_{n=0}^{K} \rho^{\alpha n}}{\bar{F}(x) \sum_{n=0}^{K} \rho^{\alpha n}}\right| \\
=\sup _{x \in G(\rho, K)} \mid \Phi\left(-\frac{(x-y)}{\sigma y}\right) \frac{\left(\sum_{n=0}^{K} \bar{F}\left(\rho^{-n} y\right)-\bar{F}(y) \sum_{n=0}^{K} \rho^{\alpha n}\right)}{\bar{F}(x) \sum_{n=0}^{K} \rho^{\alpha n}} \\
\quad+\frac{(1 /(\sigma y)) \int_{y}^{\infty} \phi(-(x-t) /(\sigma y))\left(\sum_{n=0}^{K} \bar{F}\left(\rho^{-n} t\right)-\bar{F}(t) \sum_{n=0}^{K} \rho^{\alpha n}\right) \mathrm{d} t}{\bar{F}(x) \sum_{n=0}^{K} \rho^{\alpha n}} \mid \\
\leq \sup _{x \in G(\rho, K)}\left|\frac{J(x, y)}{\bar{F}(x)}\right| \sup _{t \geq y}\left|\frac{\sum_{n=0}^{K} \bar{F}\left(\rho^{-n} t\right)-\bar{F}(t) \sum_{n=0}^{K} \rho^{\alpha n}}{\bar{F}(t) \sum_{n=0}^{K} \rho^{\alpha n}}\right|,
\end{aligned}
$$

which converges to 0 by Lemma A.1 and the observation that $J(x, y) \sim \bar{F}(x)$ uniformly for $x \in G(\rho, K)$, as proven above. This completes the proof.

With asymptotics for the partial sums $Y_{K}$ we can now give a proof of our main result, Theorem 2.1. The idea of the proof is to choose a large enough $K$ and make sure that the tail $\sum_{n=K+1}^{\infty} \rho^{i} X_{i}$ is not contributing significantly to the asymptotics of $Y_{\infty}$.

Proof of Theorem 2.1. Let $Y_{K}=\sum_{n=0}^{K} \rho^{n} X_{n}$ and $T_{K}=\sum_{n=K+1}^{\infty} \rho^{n} X_{n}$, where $K=(1-$ $\rho)^{-2}$. Define $S_{K}(\rho)=\sum_{n=0}^{K} \rho^{2 n}$ and

$$
Z(x, \rho, K)=\Phi\left(-\frac{x}{\sigma \sqrt{S_{K}(\rho)}}\right)+1\left(x \geq S_{K}(\rho)^{1 / 2}\right) \bar{F}(x) \sum_{n=0}^{K} \rho^{\alpha n} .
$$

Then, by Theorem 2.2,

$$
\mathrm{P}\left(Y_{K}>x\right)=Z(x, \rho, K)(1+o(1))
$$

as $\rho \nearrow 1$, uniformly for $x \in D(\rho, K):=\left\{x: x \leq S_{K}(\rho)^{1 / 2}\left(\log S_{K}(\rho)\right)^{1 / 2} \log \log S_{K}(\rho)\right\}$. It can be verified that

$$
Z(x, \rho, K)=\bar{F}(x) \sum_{n=0}^{K} \rho^{\alpha n}(1+o(1))
$$

as $\rho \nearrow 1$, uniformly for $x \in C(\rho, K):=\left\{x: x \geq S_{K}(\rho)^{1 / 2}\left(\log S_{K}(\rho)\right)^{1 / 2} \log \log S_{K}(\rho)\right\}$, so, by Theorem 2.3 ,

$$
\mathrm{P}\left(Y_{K}>x\right)=Z(x, \rho, K)(1+o(1))
$$

as $\rho \nearrow 1$, uniformly for $x \in \mathbb{R}$. Define

$$
Z(x, \rho)=\Phi\left(-\frac{x \sqrt{1-\rho^{2}}}{\sigma}\right)+1\left(x \geq(1-\rho)^{-1 / 2}\right)\left(1-\rho^{\alpha}\right)^{-1} \bar{F}(x) .
$$


By Lemma A.5, our choice of $K$ gives $\mathrm{P}\left(Y_{K}>x\right)=Z(x, \rho, K)(1+o(1))=Z(x, \rho)(1+o(1))$ uniformly for $x \in \mathbb{R}$. Then, for any $x \in \mathbb{R}$,

$$
\begin{aligned}
\mathrm{P}\left(Y_{\infty}>x\right) & =\mathrm{P}\left(Y_{K}+T_{K}>x\right) \\
& =\int_{-\infty}^{\infty} \mathrm{P}\left(Y_{K}>x-t\right) \mathrm{P}\left(T_{K} \in \mathrm{d} t\right) \\
& =\int_{-\infty}^{\infty} Z(x-t, \rho) \mathrm{P}\left(T_{K} \in \mathrm{d} t\right)(1+o(1)) \\
& =\mathrm{E}\left[Z\left(x-T_{K}, \rho\right)\right](1+o(1)) .
\end{aligned}
$$

We will show that $\mathrm{E}\left[Z\left(x-T_{K}, \rho\right)\right]=Z(x, \rho)(1+o(1))$ as $\rho \nearrow 1$, uniformly for $x \in \mathbb{R}$. Let $y=(1-\rho)^{-1 / 2}$, and note that, for $x \leq y, Z(x, \rho) \geq \Phi(-\sqrt{2} / \sigma)>0$. Then, using the inequality $|\Phi(a)-\Phi(b)| \leq|a-b|$, we obtain

$$
\begin{aligned}
\sup _{x<y} \mid & \left|\frac{\mathrm{E}\left[Z\left(x-T_{K}, \rho\right)\right]-Z(x, \rho)}{Z(x, \rho)}\right| \\
& \leq \sup _{x<y} C\left(\mathrm{E}\left[\left|\Phi\left(-\frac{\left(x-T_{K}\right) \sqrt{1-\rho^{2}}}{\sigma}\right)-\Phi\left(-\frac{x \sqrt{1-\rho^{2}}}{\sigma}\right)\right|\right]+\left(1-\rho^{\alpha}\right)^{-1} \bar{F}(y)\right) \\
& \leq \sup _{x<y} C\left(\mathrm{E}\left[\left|T_{K}\right|\right] \frac{\sqrt{2}}{\sigma y}+y^{2} \bar{F}(y)\right)
\end{aligned}
$$

for some constant $C>0$. Since $\alpha>2, y^{2} \bar{F}(y) \rightarrow 0$. To see that $\mathrm{E}\left[\left|T_{K}\right|\right] y^{-1} \rightarrow 0$ as well, note that

$$
\left(\frac{\mathrm{E}\left[\left|T_{K}\right|\right]}{y}\right)^{2} \leq \frac{\mathrm{E}\left[T_{K}^{2}\right]}{y^{2}}=\frac{\operatorname{var}\left(T_{K}\right)}{y^{2}}=\frac{1}{y^{2}} \sum_{n=K+1}^{\infty} \rho^{2 n} \operatorname{var}\left(X_{n}\right) \leq \sigma^{2} \rho^{2(K+1)},
$$

where $\rho^{K} \leq \mathrm{e}^{-K(1-\rho)}=\mathrm{e}^{-y^{2}}$. To analyze the supremum for values of $x \geq y$, we split it as follows:

$$
\begin{aligned}
\sup _{x \geq y}\left|\frac{\mathrm{E}\left[Z\left(x-T_{K}, \rho\right)\right]-Z(x, \rho)}{Z(x, \rho)}\right| \leq & \sup _{x \geq y} \frac{\mathrm{E}\left[\left|Z\left(x-T_{K}, \rho\right)-Z(x, \rho)\right| 1\left(\left|T_{K}\right| \leq x / \log y\right)\right]}{Z(x, \rho)} \\
& +\sup _{x \geq y} \frac{\mathrm{E}\left[\left|Z\left(x-T_{K}, \rho\right)-Z(x, \rho)\right| 1\left(\left|T_{K}\right|>x / \log y\right)\right]}{Z(x, \rho)} .
\end{aligned}
$$

Let $w=w(y)=1 / \log y$. For the first term, we use the inequality $|\Phi(a)-\Phi(b)| \leq \phi(a \wedge$ b) $|a-b|$ for $a, b>0$ to obtain

$$
\begin{aligned}
\sup _{x \geq y} \frac{\mathrm{E}\left[\left|Z\left(x-T_{K}, \rho\right)-Z(x, \rho)\right| 1\left(\left|T_{K}\right| \leq x w\right)\right]}{Z(x, \rho)} \\
\leq \sup _{x \geq y}\left(\frac{\mathrm{E}\left[\left|\Phi\left(-\left(x-T_{K}\right) \sqrt{1-\rho^{2}} / \sigma\right)-\Phi\left(-x \sqrt{1-\rho^{2}} / \sigma\right)\right| 1\left(\left|T_{K}\right| \leq x w\right)\right]}{\left(1-\rho^{\alpha}\right)^{-1} \bar{F}(x)}\right. \\
+\mathrm{E}\left[\frac{\bar{F}\left(x-T_{K}\right)}{\bar{F}(x)}-1 \mid 1\left(-x w \leq T_{K} \leq(x-y) \wedge x w\right)\right] \\
\left.+\frac{\bar{F}(x) \mathrm{P}\left((x-y) \wedge x w<T_{K} \leq x w\right)}{\left(1-\rho^{\alpha}\right) \Phi\left(-x \sqrt{1-\rho^{2}} / \sigma\right)}\right)
\end{aligned}
$$




$$
\begin{aligned}
\leq \sup _{x \geq y}( & \phi\left(-\frac{(1-w) x \sqrt{1-\rho^{2}}}{\sigma}\right) \mathrm{E}\left[\left|T_{K}\right|\right] \frac{\sqrt{1-\rho^{2}}\left(1-\rho^{\alpha}\right)}{\sigma \bar{F}(x)} \\
& \left.+\max \left\{\left|\frac{\bar{F}(x-x w)}{\bar{F}(x)}-1\right|,\left|\frac{\bar{F}(x+x w)}{\bar{F}(x)}-1\right|\right\}+\frac{y^{2} \bar{F}(x) 1(x(1-w)<y)}{\Phi\left(-x \sqrt{1-\rho^{2}} / \sigma\right)}\right) .
\end{aligned}
$$

The maximum in (3.12) converges to 0 by basic properties of regularly varying functions (since $w \rightarrow 0$ ), while the second term is of order $y^{2} \bar{F}(y)$, which also converges to 0 . Finally, the supremum in (3.11) is of order

$$
\begin{aligned}
\sup _{x \geq y} \exp \left(-\frac{x^{2}(1-w)^{2}(1+\rho)}{2 \sigma^{2} y^{2}}\right) \frac{\mathrm{E}\left[\left|T_{K}\right|\right]}{y^{3} \bar{F}(x)} \\
\quad \leq \sup _{x \geq y} \exp \left(-\frac{x^{2}(1-w)^{2}(1+\rho)}{2 \sigma^{2} y^{2}}\right) \frac{\sigma \rho^{K+1}}{y^{2} \bar{F}(x)} \quad(\text { by (3.10)) } \\
\quad \leq \sup _{t \geq 1} \exp \left(-\frac{t^{2}(1-w)^{2}(1+\rho)}{2 \sigma^{2}}\right) \frac{\bar{F}(y)}{\bar{F}(t y)} \frac{\sigma \mathrm{e}^{-y^{2}}}{y^{2} \bar{F}(y)} \\
\quad \leq \sup _{t \geq 1} \exp \left(-\frac{t^{2}(1-w)^{2}(1+\rho)}{2 \sigma^{2}}\right) A t^{-\alpha+1} \frac{\sigma \mathrm{e}^{-y^{2}}}{y^{2} \bar{F}(y)} \\
\quad \rightarrow 0,
\end{aligned}
$$

where to obtain the last inequality we used Potter's theorem (see Theorem 1.5.6(ii) of [4, p. 25]) with $\delta=1$ and $A>1$ a constant. It only remains to show that the supremum involving $\left|T_{K}\right|>x w$ converges to 0 . To do this, simply note that

$$
\begin{aligned}
\sup _{x \geq y} & \frac{\mathrm{E}\left[\left|Z\left(x-T_{K}, \rho\right)-Z(x, \rho)\right| 1\left(\left|T_{K}\right|>x w\right)\right]}{Z(x, \rho)} \\
\leq \sup _{x \geq y}\left(\frac{\mathrm{E}\left[\left|\Phi\left(-\left(x-T_{K}\right) \sqrt{1-\rho^{2}} / \sigma\right)-\Phi\left(-x \sqrt{1-\rho^{2}} / \sigma\right)\right| 1\left(\left|T_{K}\right|>x w\right)\right]}{\left(1-\rho^{\alpha}\right)^{-1} \bar{F}(x)}\right. & \left.\quad+\mathrm{E}\left[\left|\frac{\bar{F}\left(x-T_{K}\right) 1\left(T_{K} \leq x-y\right)}{\bar{F}(x)}-1\right| 1\left(\left|T_{K}\right|>x w\right)\right]\right) \\
& \leq \sup _{x \geq y}\left(\frac{\left(1-\rho^{\alpha}\right) \mathrm{P}\left(\left|T_{K}\right|>x w\right)}{\bar{F}(x)}+\left(\frac{\bar{F}(y)}{\bar{F}(x)}+1\right) \mathrm{P}\left(\left|T_{K}\right|>x w\right)\right) .
\end{aligned}
$$

Note that, for any $t \geq y w$,

$$
\begin{aligned}
\mathrm{P}\left(T_{K}>t\right) & \leq \mathrm{P}\left(\bigcup_{n=K+1}^{\infty}\left\{\rho^{n} X_{n}>t(1-\sqrt{\rho}) \rho^{(n-K-1) / 2}\right\}\right) \\
& \leq \sum_{m=0}^{\infty} \bar{F}\left(t(1-\sqrt{\rho}) \rho^{-K-1} \rho^{-m / 2}\right) \\
& =O\left(\bar{F}\left(t(1-\sqrt{\rho}) \rho^{-K-1}\right) \sum_{m=0}^{\infty} \rho^{\alpha m / 2}\right) \\
& =O\left(\bar{F}\left(t(1-\sqrt{\rho}) \rho^{-K-1}\right)\left(1-\rho^{\alpha / 2}\right)^{-1}\right) .
\end{aligned}
$$


Using Potter's theorem (see Theorem 1.5.6(ii) of [4, p. 25]) with $\delta=\alpha-2$, we obtain

$$
\mathrm{P}\left(T_{K}>t\right)=O\left(\bar{F}(t)(1-\sqrt{\rho})^{-2} \rho^{2(K+1)}\left(1-\rho^{\alpha / 2}\right)^{-1}\right)=O\left(\bar{F}(t) \mathrm{e}^{-2 y^{2}} y^{6}\right) .
$$

It follows that (3.13) is of order

$$
\sup _{x \geq y}\left(\frac{\bar{F}(x w) \mathrm{e}^{-2 y^{2}} y^{4}}{\bar{F}(x)}+\frac{\bar{F}(y) \bar{F}(x w) \mathrm{e}^{-2 y^{2}} y^{6}}{\bar{F}(x)}\right)=O\left(w^{-\alpha-\delta} \mathrm{e}^{-2 y^{2}} y^{4}\right)=o(1) .
$$

This completes the proof.

The last result of this section gives the precise threshold at which the behavior of $\mathrm{P}\left(Y_{\infty}>x\right)$ transitions from the normal approximation into the heavy-tailed asymptotic. Just as the results obtained for the M/G/1 queue in [19], the transition is very sharp and depends on the slowly varying part of $F$.

Lemma 3.1. Let

$$
Z(x, \rho)=\Phi\left(-\frac{x \sqrt{1-\rho^{2}}}{\sigma}\right)+1\left(x \geq(1-\rho)^{-1 / 2}\right)\left(1-\rho^{\alpha}\right)^{-1} \bar{F}(x),
$$

and define $z(\rho)=\kappa(1-\rho)^{-1 / 2}|\log (1-\rho)|^{1 / 2}$, where $\kappa=\sigma \sqrt{(\alpha-2) / 2}$. Then the following assertions hold.

(a) For any $c<1$, as $\rho \nearrow 1$,

$$
\sup _{0<x \leq c z(\rho)}\left|\frac{Z(x, \rho)}{\Phi\left(-x \sqrt{1-\rho^{2}} / \sigma\right)}-1\right| \rightarrow 0 .
$$

Furthermore, if $c=1$, (3.14) holds provided that $\sup _{0 \leq t \leq x} L(t) /(\log x)^{(\alpha-1) / 2} \rightarrow 0$.

(b) For any $c>1$, as $\rho \nearrow 1$,

$$
\sup _{x \geq c z(\rho)}\left|\frac{Z(x, \rho)}{\left(1-\rho^{\alpha}\right)^{-1} \bar{F}(x)}-1\right| \rightarrow 0 .
$$

Furthermore, if $c=1$, (3.15) holds provided that $\inf _{t \geq x} L(t) /(\log x)^{(\alpha-1) / 2} \rightarrow \infty$.

Proof. We start with part (a), for which we need to prove that

$$
\sup _{(1-\rho)^{-1 / 2} \leq x \leq c z(\rho)} \frac{\left(1-\rho^{\alpha}\right)^{-1} \bar{F}(x)}{\Phi\left(-x \sqrt{1-\rho^{2}} / \sigma\right)} \rightarrow 0 .
$$

Let $y=y(\rho)=(1-\rho)^{-1 / 2}$, and note that $z=z(\rho)=\kappa y\left(\log y^{2}\right)^{1 / 2}$. Use the inequality $\Phi(-z) \geq z \phi(z) /\left(z^{2}+1\right)$ to obtain

$$
\begin{aligned}
\sup _{y \leq x \leq c z} & \frac{\left(1-\rho^{\alpha}\right)^{-1} \bar{F}(x)}{\Phi\left(-x \sqrt{1-\rho^{2}} / \sigma\right)} \\
\leq & \sup _{y \leq x \leq c z} \frac{(1-\rho)^{-1} \bar{F}(x)\left(x^{2}(1-\rho)+1\right)}{x(1-\rho)^{1 / 2} \phi\left(-x \sqrt{1-\rho^{2}} / \sigma\right)} \\
\leq & \sup _{y \leq x \leq c z} C^{\prime} y L(x) \exp \left(\frac{x^{2}}{\sigma^{2} y^{2}}-(\alpha-1) \log x\right) \\
\leq & C^{\prime} y \sup _{y \leq t \leq c z} L(t) \max \left\{\exp \left(\frac{1}{\sigma^{2}}-(\alpha-1) \log y\right), \exp \left(\frac{c^{2} z^{2}}{\sigma^{2} y^{2}}-(\alpha-1) \log (c z)\right)\right\}
\end{aligned}
$$




$$
\begin{aligned}
& \leq C^{\prime \prime} y \sup _{y \leq t \leq c z} L(t) \max \left\{y^{-(\alpha-1)}, \exp \left(\frac{c^{2} \kappa^{2} \log y^{2}}{\sigma^{2}}-(\alpha-1) \log \left(y(\log y)^{1 / 2}\right)\right)\right\} \\
& =C^{\prime \prime} \sup _{y \leq t \leq c z} L(t) \max \left\{y^{-(\alpha-2)}, \frac{y^{-\left(1-c^{2}\right)(\alpha-2)}}{(\log y)^{(\alpha-1) / 2}}\right\} .
\end{aligned}
$$

That the expression above converges to 0 when $c<1$ follows from the fact that $\tilde{L}(x)=$ $\sup _{0 \leq t \leq x} L(t)$ is slowly varying. When $c=1$, just note that

$$
\frac{1}{(\log y)^{(\alpha-1) / 2}} \sup _{y \leq t \leq z} L(t) \sim \frac{1}{(\log z)^{(\alpha-1) / 2}} \sup _{y \leq t \leq z} L(t) \leq \frac{\tilde{L}(z)}{(\log z)^{(\alpha-1) / 2}} .
$$

For part (b), we need to prove that

$$
\sup _{x \geq c z(\rho)} \frac{\Phi\left(-x \sqrt{1-\rho^{2}} / \sigma\right)}{\left(1-\rho^{\alpha}\right)^{-1} \bar{F}(x)} \rightarrow 0 .
$$

We use the inequality $\Phi(-z) \leq \phi(z) / z$ to obtain

$$
\begin{aligned}
\sup _{x \geq c z} \frac{\Phi\left(-x \sqrt{1-\rho^{2}} / \sigma\right)}{\left(1-\rho^{\alpha}\right)^{-1} \bar{F}(x)} & \leq \sup _{x \geq c z} \frac{C \phi\left(x \sqrt{1-\rho^{2}} / \sigma\right)}{x(1-\rho)^{-1 / 2} \bar{F}(x)} \\
& \leq C^{\prime} \sup _{x \geq c z} \frac{1}{y L(x)} \exp \left(-\frac{x^{2}(1+\rho)}{2 \sigma^{2} y^{2}}+(\alpha-1) \log x\right) \\
& \leq \frac{C^{\prime}}{y \inf _{t \geq c z} L(t)} \exp \left(-\frac{c^{2} z^{2}(1+\rho)}{2 \sigma^{2} y^{2}}+(\alpha-1) \log (c z)\right) \\
& \leq \frac{C^{\prime \prime}}{\inf _{t \geq c z} L(t)} y^{-\left(c^{2}-1\right)(\alpha-2)}(\log y)^{(\alpha-1) / 2} .
\end{aligned}
$$

The same arguments used above give the result.

\section{Appendix A. Technical lemmas}

The first two results, Lemmas A.1 and A.2, refer to properties of regularly varying functions that are used on multiple occasions in the proofs above, and are potentially of independent interest.

Lemma A.1. As $x \rightarrow \infty$,

$$
\sup _{0<\rho<1} \sup _{K \geq 0}\left|\frac{\sum_{n=0}^{K} \bar{F}\left(\rho^{-n} x\right)}{\bar{F}(x) \sum_{n=0}^{K} \rho^{\alpha n}}-1\right| \rightarrow 0 .
$$

Proof. Fix $M \geq 1$. Then

$$
\begin{aligned}
& \sup _{K \geq 0}\left|\frac{\sum_{n=0}^{K} \bar{F}\left(\rho^{-n} x\right)}{\bar{F}(x) \sum_{n=0}^{K} \rho^{\alpha n}}-1\right| \leq \sup _{K \geq 0} \frac{1}{\sum_{n=0}^{K} \rho^{\alpha n}} \sum_{n=0}^{K} \rho^{\alpha n}\left|\frac{\bar{F}\left(\rho^{-n} x\right)}{\rho^{\alpha n} \bar{F}(x)}-1\right| \\
& \leq \max \left\{\sup _{0 \leq K \leq M|\log \rho|^{-1}} \frac{1}{\sum_{n=0}^{K} \rho^{\alpha n}} \sum_{n=0}^{K} \rho^{\alpha n}\left|\frac{L\left(\rho^{-n} x\right)}{L(x)}-1\right|,\right. \\
&\left.\sup _{K \geq M|\log \rho|^{-1}} \frac{1}{\sum_{n=0}^{K} \rho^{\alpha n}} \sum_{n=0}^{K} \rho^{\alpha n}\left|\frac{L\left(\rho^{-n} x\right)}{L(x)}-1\right|\right\}
\end{aligned}
$$




$$
\begin{aligned}
\leq \max \left\{\sup _{1 \leq t \leq \mathrm{e}^{M}}\left|\frac{L(t x)}{L(x)}-1\right|,\right. \\
\left.\quad \sup _{K \geq M|\log \rho|^{-1}} \frac{1}{\sum_{n=0}^{M|\log \rho|^{-1}} \rho^{\alpha n}} \sum_{n=0}^{K} \rho^{\alpha n}\left|\frac{L\left(\rho^{-n} x\right)}{L(x)}-1\right|\right\} .
\end{aligned}
$$

To analyze the second term, note that, for $K \geq M|\log \rho|^{-1}$,

$$
\begin{aligned}
& \sum_{n=0}^{K} \rho^{\alpha n}\left|\frac{L\left(\rho^{-n} x\right)}{L(x)}-1\right| \\
& \quad \leq \sup _{1 \leq t \leq \mathrm{e}^{M}}\left|\frac{L(t x)}{L(x)}-1\right| \sum_{n=0}^{M|\log \rho|^{-1}} \rho^{\alpha n}+\sum_{n=M|\log \rho|^{-1}+1}^{K} \rho^{\alpha n}\left|\frac{L\left(\rho^{-n} x\right)}{L(x)}-1\right|,
\end{aligned}
$$

from which we obtain

$$
\begin{aligned}
& \sup _{K \geq 0}\left|\frac{\sum_{n=0}^{K} \bar{F}\left(\rho^{-n} x\right)}{\bar{F}(x) \sum_{n=0}^{K} \rho^{\alpha n}}-1\right| \\
& \quad \leq \sup _{1 \leq t \leq \mathrm{e}^{M}}\left|\frac{L(t x)}{L(x)}-1\right|+\sup _{K \geq M|\log \rho|^{-1}} \frac{1-\rho^{\alpha}}{1-\mathrm{e}^{-\alpha M}} \sum_{n=M|\log \rho|^{-1}+1}^{K} \rho^{\alpha n}\left|\frac{L\left(\rho^{-n} x\right)}{L(x)}-1\right| .
\end{aligned}
$$

For the last sum, fix $0<\delta<\alpha-2$ and use Potter's theorem (see Theorem 1.5.6(ii) of [4, p. 25]) to obtain, for some $A_{\delta}>1$,

$$
\begin{aligned}
\sum_{n=M|\log \rho|^{-1}+1}^{K} \rho^{\alpha n}\left|\frac{L\left(\rho^{-n} x\right)}{L(x)}-1\right| & \leq \sum_{n=M|\log \rho|^{-1}+1}^{K} \rho^{\alpha n}\left(\frac{L\left(\rho^{-n} x\right)}{L(x)}+1\right) \\
& \leq A_{\delta} \sum_{n=M|\log \rho|^{-1}+1}^{\infty} \rho^{(\alpha-\delta) n}+\sum_{n=M|\log \rho|^{-1}+1}^{\infty} \rho^{\alpha n} \\
& \leq A_{\delta} \mathrm{e}^{-(\alpha-\delta) M}\left(1-\rho^{\alpha-\delta}\right)^{-1}+\mathrm{e}^{-\alpha M}\left(1-\rho^{\alpha}\right)^{-1} \\
& \leq C\left(1-\rho^{\alpha-\delta}\right)^{-1} \mathrm{e}^{-(\alpha-\delta) M} .
\end{aligned}
$$

It follows that

$$
\begin{aligned}
\sup _{0<\rho<1} \sup _{K \geq 0}\left|\frac{\sum_{n=0}^{K} \bar{F}\left(\rho^{-n} x\right)}{\bar{F}(x) \sum_{n=0}^{K} \rho^{\alpha n}}-1\right| & \leq \sup _{0<\rho<1}\left\{\sup _{1 \leq t \leq \mathrm{e}^{M}}\left|\frac{L(t x)}{L(x)}-1\right|+\frac{1-\rho^{\alpha}}{1-\rho^{\alpha-\delta}} C^{\prime} \mathrm{e}^{-(\alpha-\delta) M}\right\} \\
& =\sup _{1 \leq t \leq \mathrm{e}^{M}}\left|\frac{L(t x)}{L(x)}-1\right|+\frac{\alpha}{\alpha-\delta} C^{\prime} \mathrm{e}^{-(\alpha-\delta) M} .
\end{aligned}
$$

Since $M \geq 1$ was arbitrary, and the supremum converges to 0 as $x \rightarrow \infty$ for any fixed $M$, the result follows.

Lemma A.2. For $0<\rho<1$ and $y>0$, define

$$
W_{n}^{(y)}=\rho^{n} X_{n} \mid \rho^{n} X_{n} \leq y \quad \text { and } \quad F_{n, y}(x)=\mathrm{P}\left(W_{n}^{(y)} \leq x\right)=\frac{F\left(\rho^{-n}(x \wedge y)\right)}{F\left(\rho^{-n} y\right)} \text {. }
$$


Then, for any $2<\beta<\alpha \wedge 3$ and any $0<\phi \leq c(\log y)^{1 / 2}(\log \log y) / y$, where $c>0$ is a constant, we have

$$
\begin{aligned}
\mathrm{E}\left[\mathrm{e}^{\phi W_{n}^{(y)}}\right] & =1+\frac{\phi^{2} \rho^{2 n} \sigma^{2}}{2}+o\left(\rho^{n \beta} y^{-\beta}\right), \\
\mathrm{E}\left[W_{n}^{(y)} \mathrm{e}^{\phi W_{n}^{(y)}}\right] & =\phi \rho^{2 n} \sigma^{2}+o\left(\rho^{n \beta} y^{1-\beta}\right), \\
\mathrm{E}\left[\left(W_{n}^{(y)}\right)^{2} \mathrm{e}^{\phi W_{n}^{(y)}}\right] & =\rho^{2 n} \sigma^{2}+o\left(\rho^{n \beta} y^{2-\beta}\right),
\end{aligned}
$$

as $y \rightarrow \infty$.

Proof. We start by noting that the balance condition satisfied by $F$ guarantees that $\mathrm{E}\left[\left|X_{1}\right|^{\gamma}\right]<\infty$ for any $0 \leq \gamma<\alpha$. Choose $\beta<\gamma<\min \{\alpha, 3\}$. The first step of the analysis is to write

$$
\begin{aligned}
\mathrm{E}\left[\mathrm{e}^{\phi W_{n}^{(y)}}\right]= & 1+\phi \mathrm{E}\left[W_{n}^{(y)}\right]+\frac{\phi^{2}}{2} \mathrm{E}\left[\left(W_{n}^{(y)}\right)^{2}\right] \\
& +\mathrm{E}\left[\mathrm{e}^{\phi W_{n}^{(y)}}-1-\phi W_{n}^{(y)}-\frac{\phi^{2}}{2}\left(W_{n}^{(y)}\right)^{2}\right], \\
\mathrm{E}\left[W_{n}^{(y)} \mathrm{e}^{\phi W_{n}^{(y)}}\right]= & \mathrm{E}\left[W_{n}^{(y)}\right]+\phi \mathrm{E}\left[\left(W_{n}^{(y)}\right)^{2}\right]+\mathrm{E}\left[W_{n}^{(y)}\left(\mathrm{e}^{\phi W_{n}^{(y)}}-1-\phi W_{n}^{(y)}\right)\right], \\
\mathrm{E}\left[\left(W_{n}^{(y)}\right)^{2} \mathrm{e}^{\phi W_{n}^{(y)}}\right]= & \mathrm{E}\left[\left(W_{n}^{(y)}\right)^{2}\right]+\mathrm{E}\left[\left(W_{n}^{(y)}\right)^{2}\left(\mathrm{e}^{\phi W_{n}^{(y)}}-1\right)\right] .
\end{aligned}
$$

The first two moments of $W_{n}^{(y)}$ can be computed to be

$$
\begin{gathered}
\mathrm{E}\left[W_{n}^{(y)}\right]=\frac{\rho^{n}}{F\left(\rho^{-n} y\right)}\left(\mathrm{E}\left[X_{1}\right]-\int_{\rho^{-n} y}^{\infty} u F(\mathrm{~d} u)\right)=O\left(y \bar{F}\left(\rho^{-n} y\right)\right), \\
\mathrm{E}\left[\left(W_{n}^{(y)}\right)^{2}\right]=\frac{\rho^{2 n}}{F\left(\rho^{-n} y\right)}\left(\mathrm{E}\left[X_{1}^{2}\right]-\int_{\rho^{-n} y}^{\infty} u^{2} F(\mathrm{~d} u)\right)=\rho^{2 n} \sigma^{2}+O\left(y^{2} \bar{F}\left(\rho^{-n} y\right)\right) .
\end{gathered}
$$

Note that $\bar{F}(x)=o\left(x^{-\gamma}\right)$ as $x \rightarrow \infty$, so combining the estimates above we obtain

$$
\begin{aligned}
& \mathrm{E}\left[\mathrm{e}^{\phi W_{n}^{(y)}}\right]=1+\frac{\phi^{2} \rho^{2 n} \sigma^{2}}{2}+o\left(\phi \rho^{n \gamma} y^{1-\gamma}(1+\phi y)\right) \\
& +\mathrm{E}\left[\mathrm{e}^{\phi W_{n}^{(y)}}-1-\phi W_{n}^{(y)}-\frac{\phi^{2}}{2}\left(W_{n}^{(y)}\right)^{2}\right] \\
& \mathrm{E}\left[W_{n}^{(y)} \mathrm{e}^{\phi W_{n}^{(y)}}\right]=\phi \rho^{2 n} \sigma^{2}+o\left(\rho^{n \gamma} y^{1-\gamma}(1+\phi y)\right)+\mathrm{E}\left[W_{n}^{(y)}\left(\mathrm{e}^{\phi W_{n}^{(y)}}-1-\phi W_{n}^{(y)}\right)\right], \\
& \mathrm{E}\left[\left(W_{n}^{(y)}\right)^{2} \mathrm{e}^{\phi W_{n}^{(y)}}\right]=\rho^{2 n} \sigma^{2}+o\left(\rho^{n \gamma} y^{2-\gamma}\right)+\mathrm{E}\left[\left(W_{n}^{(y)}\right)^{2}\left(\mathrm{e}^{\phi W_{n}^{(y)}}-1\right)\right] . \\
& \mathrm{E}\left[\left|g_{2}\left(\phi W_{n}^{(y)}\right)\right|\right] \leq \frac{\phi^{3} \mathrm{e}}{6} \mathrm{E}\left[\left|W_{n}^{(y)}\right|^{3} 1\left(\left|W_{n}^{(y)}\right| \leq \frac{1}{\phi}\right)\right] \phi^{2} \mathrm{E}\left[\left(W_{n}^{(y)}\right)^{2} 1\left(W_{n}^{(y)}<-\frac{1}{\phi}\right)\right] \\
& +\frac{\phi^{3}}{6} \mathrm{E}\left[\left(W_{n}^{(y)}\right)^{3} \mathrm{e}^{\phi W_{n}^{(y)}} 1\left(W_{n}^{(y)}>\frac{1}{\phi}\right)\right] \text {. }
\end{aligned}
$$


Similarly, using $g_{1}(t)=t^{2} \mathrm{e}^{\xi_{1}} / 2$ and $g_{0}(t)=t \mathrm{e}^{\xi_{3}}$, we obtain

$$
\begin{aligned}
\mathrm{E}\left[\left|W_{n}^{(y)} g_{1}\left(\phi W_{n}^{(y)}\right)\right|\right] \leq & \frac{\phi^{2} \mathrm{e}}{2} \mathrm{E}\left[\left|W_{n}^{(y)}\right|^{3} 1\left(\left|W_{n}^{(y)}\right| \leq \frac{1}{\phi}\right)\right]+2 \phi \mathrm{E}\left[\left(W_{n}^{(y)}\right)^{2} 1\left(W_{n}^{(y)}<-\frac{1}{\phi}\right)\right] \\
& +\frac{\phi^{2}}{2} \mathrm{E}\left[\left(W_{n}^{(y)}\right)^{3} 1\left(W_{n}^{(y)}>\frac{1}{\phi}\right)\right] .
\end{aligned}
$$

Finally,

$$
\begin{aligned}
\mathrm{E}\left[\left|\left(W_{n}^{(y)}\right)^{2} g_{0}\left(\phi W_{n}^{(y)}\right)\right|\right] \leq & \phi \mathrm{e} \mathrm{E}\left[\left|W_{n}^{(y)}\right|^{3} 1\left(\left|W_{n}^{(y)}\right| \leq \frac{1}{\phi}\right)\right]+\mathrm{E}\left[\left(W_{n}^{(y)}\right)^{2} 1\left(W_{n}^{(y)}<-\frac{1}{\phi}\right)\right] \\
& +\phi \mathrm{E}\left[\left(W_{n}^{(y)}\right)^{3} \mathrm{e}^{\phi W_{n}^{(y)}} 1\left(W_{n}^{(y)}>\frac{1}{\phi}\right)\right] .
\end{aligned}
$$

We now analyze each of the three expectations appearing in the expressions above. First, note that

$$
\begin{aligned}
\mathrm{E}\left[\left|W_{n}^{(y)}\right|^{3} 1\left(\left|W_{n}^{(y)}\right| \leq \frac{1}{\phi}\right)\right] & =\rho^{3 n} \int_{-\infty}^{\rho^{-n} y}|x|^{3} 1\left(\left|\rho^{n} x\right| \leq \frac{1}{\phi}\right) \frac{F(\mathrm{~d} x)}{F\left(\rho^{-n} y\right)} \\
& =\frac{\rho^{3 n}}{F\left(\rho^{-n} y\right)} \int_{-\rho^{-n} / \phi}^{\rho^{-n}(y \wedge 1 / \phi)}|x|^{3} F(\mathrm{~d} x) \\
& \leq \frac{\rho^{3 n}\left(\rho^{-n} / \phi\right)^{3-\gamma}}{F\left(\rho^{-n} y\right)} \int_{-\rho^{-n} / \phi}^{\rho^{-n}(y \wedge 1 / \phi)}|x|^{\gamma} F(\mathrm{~d} x) \\
& \leq \frac{\rho^{n \gamma} \phi^{-(3-\gamma)}}{F(y)} \mathrm{E}\left[\left|X_{1}\right|^{3}\right] \\
& =O\left(\rho^{n \gamma} \phi^{\gamma-3}\right) .
\end{aligned}
$$

Next, we bound the second expectation as follows:

$$
\begin{aligned}
\mathrm{E}\left[\left(W_{n}^{(y)}\right)^{2} 1\left(W_{n}^{(y)}<-\frac{1}{\phi}\right)\right] & =\rho^{2 n} \int_{-\infty}^{\rho^{-n} y} x^{2} 1\left(\rho^{n} x<-\frac{1}{\phi}\right) \frac{F(\mathrm{~d} x)}{F\left(\rho^{-n} y\right)} \\
& =\frac{\rho^{2 n}}{F\left(\rho^{-n} y\right)}\left(\rho^{-2 n} F\left(-\frac{\rho^{-n}}{\phi}\right)-\int_{-\infty}^{-\rho^{-n} / \phi} 2 x F(x) \mathrm{d} x\right) \\
& =O\left(\bar{F}\left(\frac{\rho^{-n}}{\phi}\right)+\rho^{2 n} \int_{\rho^{-n} / \phi}^{\infty} x \bar{F}(x) \mathrm{d} x\right) \\
& =O\left(\phi^{-2} \bar{F}\left(\frac{\rho^{-n}}{\phi}\right)\right) \\
& =o\left(\rho^{n \gamma} \phi^{\gamma-2}\right),
\end{aligned}
$$

where in the third equality we used the balance condition satisfied by $F$ and in the fourth equality we used Karamata's theorem. To analyze the last expectation, recall that $W_{n}^{(y)} \leq y$, so

$$
\begin{aligned}
\mathrm{E}\left[\left(W_{n}^{(y)}\right)^{3} \mathrm{e}^{\phi W_{n}^{(y)}} 1\left(W_{n}^{(y)}>\frac{1}{\phi}\right)\right] & \leq \mathrm{e}^{\phi y} \mathrm{E}\left[\left(W_{n}^{(y)}\right)^{3} 1\left(W_{n}^{(y)}>\frac{1}{\phi}\right)\right] \\
& =\mathrm{e}^{\phi y} \rho^{3 n} \int_{-\infty}^{\rho^{-n} y} x^{3} 1\left(\rho^{n} x>\frac{1}{\phi}\right) \frac{F(\mathrm{~d} x)}{F\left(\rho^{-n} y\right)}
\end{aligned}
$$




$$
\begin{aligned}
& \leq \frac{\mathrm{e}^{\phi y} \rho^{3 n}\left(\rho^{-n} y\right)^{3-\gamma}}{F\left(\rho^{-n} y\right)} \int_{\rho^{-n}(y \wedge 1 / \phi)}^{\rho^{-n} y} x^{\gamma} \mathrm{d} x \\
& \leq \frac{\mathrm{e}^{\phi y} \rho^{n \gamma} y^{3-\gamma} \mathrm{E}\left[\left|X_{1}\right|^{\gamma}\right]}{F\left(\rho^{-n} y\right)} \\
& =O\left(\phi^{\gamma-3} \rho^{n \gamma} \mathrm{e}^{\phi y}(\phi y)^{3-\gamma}\right) .
\end{aligned}
$$

Note that, for the range of values of $\phi$ that we consider, we have

$$
\mathrm{e}^{\theta y}(\theta y)^{3-\gamma} \leq \mathrm{e}^{c(\log y)^{1 / 2}(\log \log y)}\left(c(\log y)^{1 / 2}(\log \log y)\right)^{3-\gamma}=: \hat{L}(y),
$$

where $\hat{L}(y) \rightarrow \infty$ is slowly varying. Combining the estimates derived above we obtain

$$
\begin{aligned}
\mathrm{E}\left[W_{n}^{(y)}\left(\mathrm{e}^{\phi W_{n}^{(y)}}-1-\phi W_{n}^{(y)}\right)\right] & =O\left(\phi^{\gamma} \rho^{n \gamma} \hat{L}(y)\right), \\
\mathrm{E}\left[W_{n}^{(y)}\left(\mathrm{e}^{\phi W_{n}^{(y)}}-1-\phi W_{n}^{(y)}\right)\right] & =O\left(\phi^{\gamma-1} \rho^{n \gamma} \hat{L}(y)\right), \\
\mathrm{E}\left[\left(W_{n}^{(y)}\right)^{2}\left(\mathrm{e}^{\phi W_{n}^{(y)}}-1\right)\right] & =O\left(\phi^{\gamma-2} \rho^{n \gamma} \hat{L}(y)\right),
\end{aligned}
$$

which in turn yield

$$
\begin{aligned}
\mathrm{E}\left[\mathrm{e}^{\phi W_{n}^{(y)}}\right] & =1+\frac{\phi^{2} \rho^{2 n} \sigma^{2}}{2}+o\left(\phi \rho^{n \gamma} y^{1-\gamma}(1+\phi y)\right)+O\left(\phi^{\gamma} \rho^{n \gamma} \hat{L}(y)\right), \\
\mathrm{E}\left[W_{n}^{(y)} \mathrm{e}^{\phi W_{n}^{(y)}}\right] & =\phi \rho^{2 n} \sigma^{2}+o\left(\rho^{n \gamma} y^{1-\gamma}(1+\phi y)\right)+O\left(\phi^{\gamma-1} \rho^{n \gamma} \hat{L}(y)\right), \\
\mathrm{E}\left[\left(W_{n}^{(y)}\right)^{2} \mathrm{e}^{\phi W_{n}^{(y)}}\right] & =\rho^{2 n} \sigma^{2}+o\left(\rho^{n \gamma} y^{2-\gamma}\right)+O\left(\phi^{\gamma-2} \rho^{n \gamma} \hat{L}(y)\right) .
\end{aligned}
$$

The statement of the lemma follows by noting that $\rho^{n \gamma} \leq \rho^{n \beta}$ and $\phi \leq y^{-1} \tilde{L}(y)$, where $\tilde{L}$ is slowly varying, and using the fact that $y^{\beta-\gamma} L_{0}(y) \rightarrow 0$ for any slowly varying $L_{0}$.

The following two results, Lemmas A.3 and A.4, give asymptotic expressions for the change of measure parameter and the moments of the resulting tilted random variables used in the proof of Proposition 3.1.

Lemma A.3. Set $y=y(\rho, K)=S_{K}(\rho)^{1 / 2}$, where $S_{K}(\rho)=\sum_{n=0}^{K} \rho^{2 n}$ and $K \rightarrow \infty$ as $\rho \nearrow 1$. Let $W_{i}^{(y)}=\rho^{i} X_{i} \mid \rho^{i} X_{i} \leq y, F_{i, y}(x)=\mathrm{P}\left(W_{i}^{(y)} \leq x\right)=F\left(\rho^{-i}(x \wedge y)\right) / F\left(\rho^{-i} y\right)$, and $\Lambda_{i, y}(\theta)=\log \mathrm{E}\left[\mathrm{e}^{\theta W_{i}^{(y)}}\right]$. Define $\hat{\theta}=\hat{\theta}(\rho, K, u, n)$ to be the positive solution to the equation

$$
-u+\sum_{i \in I(K, n)} \Lambda_{i, y}^{\prime}(\hat{\theta})=0,
$$

where $I(K, n)=\{i: 0 \leq i \leq K, i \neq n\}$. Then, for any $0 \leq n \leq K$ and $0<\gamma<(\alpha-2) \wedge 1$, and, as $\rho \nearrow 1$,

$$
\hat{\theta}(\rho, K, u, n)=\frac{u}{\sigma^{2} S_{K}(\rho)}+o\left(\left(\frac{u}{S_{K}(\rho)}\right)^{1+\gamma}\right)
$$

uniformly for $u \in B(\rho, K)=\left\{x: S_{K}(\rho)^{1 / 2} \leq x \leq S_{K}(\rho)^{1 / 2}\left(\log S_{K}(\rho)\right)^{1 / 2} \log \log S_{K}(\rho)\right\}$.

Proof. We start by noting that the balance condition satisfied by $F$ guarantees that $\mathrm{E}\left[\left|X_{1}\right|^{\beta}\right]<\infty$ for any $0 \leq \beta<\alpha$. Choose $2+\gamma<\beta<\alpha \wedge 3$. Fix $0<\varepsilon<1$, and define

$$
\theta_{ \pm \varepsilon}=\frac{u}{\sigma^{2} S_{K}(\rho)}\left(1 \pm \varepsilon\left(\frac{u}{S_{K}(\rho)}\right)^{\gamma}\right) .
$$


We will show that

$$
-u+\sum_{i \in I(K, n)} \Lambda_{i, y}^{\prime}\left(\theta_{-\varepsilon}\right)<0 \text { and }-u+\sum_{i \in I(K, n)} \Lambda_{i, y}^{\prime}\left(\theta_{+\varepsilon}\right)>0 .
$$

To simplify the notation, let $S=S_{K}(\rho)$ and recall that $K \rightarrow \infty$, so $S \rightarrow \infty$ as $\rho \nearrow 1$. Note that $y \rightarrow \infty$ as well, and, for $u \in B(\rho, K)$,

$$
\begin{aligned}
\theta_{ \pm \varepsilon} & \leq \frac{(\log S)^{1 / 2} \log \log S}{\sigma^{2} S^{1 / 2}}\left(1+\varepsilon\left(\frac{(\log S)^{1 / 2} \log \log S}{S^{1 / 2}}\right)^{\gamma}\right) \\
& =\frac{(2 \log y)^{1 / 2} \log (2 \log y)}{\sigma^{2} y}\left(1+\varepsilon\left(\frac{(2 \log y)^{1 / 2} \log (2 \log y)}{y}\right)^{\gamma}\right) \\
& \leq \frac{C(\log y)^{1 / 2}(\log \log y)}{y}
\end{aligned}
$$

for some constant $C>0$. Therefore, by Lemma A.2,

$$
\begin{aligned}
\sum_{i \in I(K, n)} \Lambda_{i, y}^{\prime}\left(\theta_{ \pm \varepsilon}\right) & =\sum_{i \in I(K, n)} \frac{\mathrm{E}\left[W_{i}^{(y)} \mathrm{e}^{\left.\theta_{ \pm \varepsilon} W_{i}^{(y)}\right]}\right.}{\mathrm{E}\left[\mathrm{e}^{\theta_{ \pm \varepsilon} W_{i}^{(y)}}\right]} \\
& =\sum_{i \in I(K, n)} \frac{\theta_{ \pm \varepsilon} \rho^{2 i} \sigma^{2}+o\left(\rho^{i \beta} y^{1-\beta}\right)}{1+\theta_{ \pm \varepsilon}^{2} \rho^{2 i} \sigma^{2} / 2+o\left(\rho^{i \beta} y^{-\beta}\right)} \\
& =\sum_{i \in I(K, n)}\left[\theta_{ \pm \varepsilon} \rho^{2 i} \sigma^{2}+O\left(\theta_{ \pm \varepsilon}^{3} \rho^{4 i}\right)+o\left(\rho^{i \beta} y^{1-\beta}\right)\right] \\
& =\theta_{ \pm \varepsilon} \sigma^{2}\left(S-\rho^{2 n}\right)+O\left(\theta_{ \pm \varepsilon}^{3} \sum_{i=0}^{K} \rho^{4 i}\right)+o\left(y^{1-\beta} \sum_{i=0}^{K} \rho^{\beta i}\right) \\
& =\theta_{ \pm \varepsilon} \sigma^{2} S+O\left(\theta_{ \pm \varepsilon}+\theta_{ \pm \varepsilon}^{3} S\right)+o\left(S^{(3-\beta) / 2}\right) .
\end{aligned}
$$

Substituting the definition of $\theta_{ \pm \varepsilon}$ into the expression above and noting that $\theta_{ \pm \varepsilon}=O(u / S)$, gives

$$
\begin{aligned}
\sum_{i \in I(K, n)} \Lambda_{i, y}^{\prime}\left(\theta_{ \pm \varepsilon}\right) & =u\left(1 \pm \varepsilon\left(\frac{u}{S}\right)^{\gamma}\right)+O\left(\left(\frac{u}{S}\right)+\left(\frac{u}{S}\right)^{3} S\right)+o\left(S^{(3-\beta) / 2}\right) \\
& =u\left(1 \pm \varepsilon\left(\frac{u}{S}\right)^{\gamma}+O\left(\left(\frac{1}{S}\right)+\left(\frac{u}{S}\right)^{2}\right)+o\left(\frac{S^{(3-\beta) / 2}}{u}\right)\right) \\
& =u\left(1 \pm\left(\frac{u}{S}\right)^{\gamma}\left[\varepsilon+o\left(\frac{1}{S^{(\beta-2-\gamma) / 2}}\right)\right]\right)
\end{aligned}
$$

where in the last step we used the fact that $S^{1 / 2} \leq u \leq S^{1 / 2}(\log S)^{1 / 2} \log \log S$. Therefore,

$$
-u+\sum_{i \in I(K, n)} \Lambda_{i, y}^{\prime}\left(\theta_{ \pm \varepsilon}\right)= \pm u\left(\frac{u}{S}\right)^{\gamma}(\varepsilon+o(1)) .
$$

Since $\varepsilon>0$ was arbitrary, the statement of the lemma follows. 
Lemma A.4. Let $S_{K}(\rho)=\sum_{n=0}^{K} \rho^{2 n}, y=y(\rho, K)=S_{K}(\rho)^{1 / 2}, W_{i}^{(y)}=\rho^{i} X_{i} \mid \rho^{i} X_{i} \leq y$, $F_{i, y}(x)=\mathrm{P}\left(W_{i}^{(y)} \leq x\right), \Lambda_{i, y}(\theta)=\log \mathrm{E}\left[\mathrm{e}^{\theta W_{i}^{(y)}}\right]$, and $F_{\theta, i, y}(\mathrm{~d} x)=\mathrm{e}^{\theta x-\Lambda_{i, y}(\theta)} F_{i, y}(\mathrm{~d} x)$. Suppose that $V_{i}$ is distributed according to $F_{\hat{\theta}, i, y}(\cdot)$, where $\hat{\theta}=\hat{\theta}(\rho, K, u, n)$ is the positive solution to the equation

$$
-u+\sum_{i \in I(K, n)} \Lambda_{i, y}^{\prime}(\hat{\theta})=0,
$$

where $K \rightarrow \infty$ as $\rho \nearrow 1, I(K, n)=\{i: 0 \leq i \leq K, i \neq n\}$, and

$$
u \in B(\rho, K)=\left\{x: S_{K}(\rho)^{1 / 2} \leq x \leq S_{K}(\rho)^{1 / 2}\left(\log S_{K}(\rho)\right)^{1 / 2} \log \log S_{K}(\rho)\right\} .
$$

Suppose that $\mathrm{E}\left[\left|X_{1}\right|^{2+\delta}\right]<\infty$ and $\mathrm{E}\left[X_{1}^{2}\right]=\sigma^{2}$. Let $\hat{V}_{i}=V_{i}-\mathrm{E}\left[V_{i}\right]$. Then, for any $0<\gamma<(\alpha-2) \wedge 1$,

$$
\begin{gathered}
\mathrm{E}\left[\hat{V}_{i}^{2}\right]=\rho^{2 i} \sigma^{2}+o\left(\rho^{i(2+\gamma)} y^{-\gamma}\right), \\
\mathrm{E}\left[\left|\hat{V}_{i}\right|^{2+\delta}\right]=O\left(\mathrm{e}^{\hat{\theta} y} \rho^{(2+\delta) i}\right), \\
\exp \left(\sum_{i \in I(K, n)} \Lambda_{i, y}(\hat{\theta})\right) \prod_{i \in I(K, n)} F\left(\rho^{-i} y\right)=\exp \left(\frac{u^{2}}{2 \sigma^{2} S_{K}(\rho)}+o\left(y^{-\gamma}\right)\right),
\end{gathered}
$$

uniformly for $x \in B(\rho, K)=\left\{x: S_{K}(\rho)^{1 / 2} \leq x \leq S_{K}(\rho)^{1 / 2}\left(\log S_{K}(\rho)\right)^{1 / 2} \log \log S_{K}(\rho)\right\}$.

Proof. Choose $2+\gamma<\beta<\alpha \wedge 3$. We start by noting that

$$
\begin{aligned}
\mathrm{E}\left[V_{i}\right] & =\int_{-\infty}^{y} z F_{\hat{\theta}, i, y}(\mathrm{~d} z)=\int_{-\infty}^{y} z \mathrm{e}^{\hat{\theta} z-\Lambda_{i, y}(\hat{\theta})} F_{i, y}(\mathrm{~d} z)=\frac{\mathrm{E}\left[W_{i}^{(y)} \mathrm{e}^{\hat{\theta} W_{i}^{(y)}}\right]}{\mathrm{E}\left[\mathrm{e}^{\hat{\theta} W_{i}^{(y)}}\right]}, \\
\mathrm{E}\left[V_{i}^{2}\right] & =\int_{-\infty}^{y} z^{2} F_{\hat{\theta}, i, y}(\mathrm{~d} z)=\int_{-\infty}^{y} z^{2} \mathrm{e}^{\hat{\theta} z-\Lambda_{i, y}(\hat{\theta})} F_{i, y}(\mathrm{~d} z)=\frac{\mathrm{E}\left[\left(W_{i}^{(y)}\right)^{2} \mathrm{e}^{\hat{\theta} W_{i}^{(y)}}\right]}{\mathrm{E}\left[\mathrm{e}^{\hat{\theta} W_{i}^{(y)}}\right]} .
\end{aligned}
$$

Since $\hat{\theta}=O(u / S)$, then $\hat{\theta}$ satisfies the conditions of Lemma A.2 for all $u \in B(\rho, K)$. It follows from Lemma A.2 that

$$
\begin{aligned}
\mathrm{E}\left[V_{i}\right] & =\frac{\hat{\theta} \rho^{2 i} \sigma^{2}+o\left(\rho^{i \beta} y^{1-\beta}\right)}{1+\hat{\theta}^{2} \rho^{2 i} \sigma^{2} / 2+o\left(\rho^{i \beta} y^{-\beta}\right)}=\hat{\theta} \rho^{2 i} \sigma^{2}+o\left(\rho^{i \beta} y^{1-\beta}\right), \\
\mathrm{E}\left[V_{i}^{2}\right] & =\frac{\rho^{2 i} \sigma^{2}+o\left(\rho^{i \beta} y^{2-\beta}\right)}{1+\hat{\theta}^{2} \rho^{2 i} \sigma^{2} / 2+o\left(\rho^{i \beta} y^{-\beta}\right)}=\rho^{2 i} \sigma^{2}+o\left(\rho^{i \beta} y^{2-\beta}\right) .
\end{aligned}
$$

Therefore,

$$
\mathrm{E}\left[\hat{V}_{i}^{2}\right]=\mathrm{E}\left[V_{i}^{2}\right]-\left(\mathrm{E}\left[V_{i}\right]\right)^{2}=\rho^{2 i} \sigma^{2}+o\left(\rho^{i \beta} y^{2-\beta}\right)=\rho^{2 i} \sigma^{2}+o\left(\rho^{i(2+\gamma)} y^{-\gamma}\right) .
$$

To bound the $2+\delta$ moment, note that

$$
\begin{aligned}
\mathrm{E}\left[\left|\hat{V}_{i}\right|^{2+\delta}\right] & =\mathrm{E}\left[\left|V_{i}-\mathrm{E}\left[V_{i}\right]\right|^{2+\delta}\right] \\
& =\int_{0}^{y}\left|z-\mathrm{E}\left[V_{i}\right]\right|^{2+\delta} \mathrm{e}^{\hat{\theta} z-\Lambda_{i, y}(\hat{\theta})} F_{i, y}(\mathrm{~d} z) \\
& \leq \frac{\mathrm{e}^{\hat{\theta} y}}{\mathrm{E}\left[\mathrm{e}^{\hat{\theta} W_{i}^{(y)}}\right]} \mathrm{E}\left[\left|W_{i}^{(y)}-\mathrm{E}\left[V_{i}\right]\right|^{2+\delta}\right]
\end{aligned}
$$




$$
\begin{aligned}
& =\frac{\mathrm{e}^{\hat{\theta} y}}{\mathrm{E}\left[\mathrm{e}^{\hat{\theta} W_{i}^{(y)}}\right] F\left(\rho^{-i} y\right)} \mathrm{E}\left[\left|\rho^{i} X_{i}-\mathrm{E}\left[V_{i}\right]\right|^{2+\delta} 1\left(\rho^{i} X_{i} \leq y\right)\right] \\
& \leq \mathrm{e}^{\hat{\theta} y} \mathrm{E}\left[\left|\rho^{i} X_{i}+O\left(\hat{\theta} \rho^{2 i}\right)\right|^{2+\delta}\right] \\
& =O\left(\mathrm{e}^{\hat{\theta} y} \rho^{(2+\delta) i}\right) .
\end{aligned}
$$

Finally, for the third statement of the lemma, we use Lemma A.2 again to obtain

$$
\begin{aligned}
& \exp \left(\sum_{i \in I(K, n)} \Lambda_{i, y}(\hat{\theta})\right) \prod_{i \in I(K, n)} F\left(\rho^{-i} y\right) \\
& =\prod_{i \in I(K, n)} \mathrm{E}\left[\mathrm{e}^{\left.\hat{\theta} W_{i}^{(y)}\right] F\left(\rho^{-i} y\right)}\right. \\
& =\prod_{i \in I(K, n)}\left(1+\frac{\hat{\theta}^{2} \rho^{2 i} \sigma^{2}}{2}+o\left(\rho^{i \beta} y^{-\beta}\right)\right)\left(1-\bar{F}\left(\rho^{-i} y\right)\right) \\
& =\prod_{i \in I(K, n)}\left(1+\frac{\hat{\theta}^{2} \rho^{2 i} \sigma^{2}}{2}+o\left(\rho^{i \beta} y^{-\beta}\right)\right) \\
& =\exp \left(\sum_{i \in I(K, n)} \log \left(1+\frac{\hat{\theta}^{2} \rho^{2 i} \sigma^{2}}{2}+o\left(\rho^{i \beta} y^{-\beta}\right)\right)\right)
\end{aligned}
$$

Using the identity $\log (1+t)=t+O\left(t^{2}\right)$ and noting that $\hat{\theta}^{2}=o\left(y^{-\beta}\right)$ gives

$$
\begin{aligned}
\sum_{i \in I(K, n)} \log \left(1+\frac{\hat{\theta}^{2} \rho^{2 i} \sigma^{2}}{2}+o\left(\rho^{i \beta} y^{-\beta}\right)\right) & =\sum_{i \in I(K, n)}\left(\frac{\hat{\theta}^{2} \rho^{2 i} \sigma^{2}}{2}+o\left(\rho^{i \beta} y^{-\beta}\right)\right) \\
& =\frac{\hat{\theta}^{2} \sigma^{2}}{2}\left(S_{K}(\rho)-\rho^{2 n}\right)+o\left(y^{-\beta} \sum_{i=0}^{K} \rho^{i \beta}\right) \\
& =\frac{\hat{\theta}^{2} \sigma^{2}}{2} S_{K}(\rho)+o\left(y^{-\beta} S_{K}(\rho)\right) .
\end{aligned}
$$

Finally, by Lemma A.3,

$$
\begin{aligned}
& \frac{\hat{\theta}^{2} \sigma^{2}}{2} S_{K}(\rho)+o\left(y^{-\beta} S_{K}(\rho)\right) \\
& \quad=\left(\frac{u}{\sigma^{2} S_{K}(\rho)}+o\left(\left(\frac{u}{S_{K}(\rho)}\right)^{\beta-1}\right)\right)^{2} \frac{\sigma^{2}}{2} S_{K}(\rho)+o\left(y^{-\beta} S_{K}(\rho)\right) \\
& \quad=\frac{u^{2}}{2 \sigma^{2} S_{K}(\rho)}+o\left(\left(\frac{u}{S_{K}(\rho)}\right)^{\beta} S_{K}(\rho)\right)
\end{aligned}
$$

where the last step follows by noting that $u / S_{K}(\rho) \geq y^{-1}$ for $u \in B(\rho, K)$. Noting that

$$
\left(\frac{u}{S_{K}(\rho)}\right)^{\beta} S_{K}(\rho) \leq \frac{\left(\log S_{K}(\rho)\right)^{\beta / 2}\left(\log \log S_{K}(\rho)\right)^{\beta}}{S_{K}(\rho)^{(\beta-2) / 2}}=o\left(\frac{1}{S_{K}(\rho)^{\gamma / 2}}\right)=o\left(y^{-\gamma}\right)
$$

completes the proof. 
The last result shows how large $K$ must be so that the tail $\sum_{n=K+1}^{\infty} \rho^{n} X_{n}$ becomes negligible in the asymptotic behavior of $Y_{\infty}$.

Lemma A.5. Let $K \geq(1-\rho)^{-2}, S_{K}(\rho)=\sum_{n=0}^{K} \rho^{2 n}$,

$$
Z(x, \rho, K)=\Phi\left(-\frac{x}{\sigma \sqrt{S_{K}(\rho)}}\right)+1\left(x \geq S_{K}(\rho)^{1 / 2}\right) \bar{F}(x) \sum_{n=0}^{K} \rho^{\alpha n},
$$

and

$$
Z(x, \rho)=\Phi\left(-\frac{x \sqrt{1-\rho^{2}}}{\sigma}\right)+1\left(x \geq(1-\rho)^{-1 / 2}\right)\left(1-\rho^{\alpha}\right)^{-1} \bar{F}(x) .
$$

Then, as $\rho \nearrow 1$,

$$
\sup _{x \in \mathbb{R}}\left|\frac{Z(x, \rho, K)}{Z(x, \rho)}-1\right| \rightarrow 0 .
$$

Proof. We start by pointing out that our choice of $K$ gives

$$
\rho^{K}=\mathrm{e}^{K \log \rho} \sim \mathrm{e}^{-K(1-\rho)} \leq \mathrm{e}^{-(1-\rho)^{-1}} \rightarrow 0
$$

as $\rho \nearrow 1$. Also, note that, since $S_{K}(\rho)=\left(1-\rho^{2(K+1)}\right)\left(1-\rho^{2}\right)^{-1}<(1-\rho)^{-1}$,

$$
\begin{aligned}
\mid Z(x, \rho) & -Z(x, \rho, K) \mid \\
\leq & \left|\Phi\left(\frac{x \sqrt{1-\rho^{2}}}{\sigma \sqrt{1-\rho^{2(K+1)}}}\right)-\Phi\left(\frac{x \sqrt{1-\rho^{2}}}{\sigma}\right)\right| \\
& +\left|1\left(S_{K}(\rho)^{1 / 2} \leq x<(1-\rho)^{-1 / 2}\right)-\rho^{\alpha(K+1)} 1\left(x \geq S_{K}(\rho)^{1 / 2}\right)\right| \frac{\bar{F}(x)}{\left(1-\rho^{\alpha}\right)} .
\end{aligned}
$$

Since $|\Phi(a)-\Phi(b)| \leq \phi(|a| \wedge|b|)|a-b|$ whenever $a$ and $b$ have the same sign, then

$$
\begin{aligned}
& \left|\Phi\left(\frac{x \sqrt{1-\rho^{2}}}{\sigma \sqrt{1-\rho^{2(K+1)}}}\right)-\Phi\left(\frac{x \sqrt{1-\rho^{2}}}{\sigma}\right)\right| \\
& \quad \leq \phi\left(\frac{|x| \sqrt{1-\rho^{2}}}{\sigma}\right)\left|\frac{1}{\sqrt{1-\rho^{2(K+1)}}}-1\right| \frac{|x| \sqrt{1-\rho^{2}}}{\sigma} \\
& \quad \leq \phi\left(\frac{|x| \sqrt{1-\rho^{2}}}{\sigma}\right) \frac{\rho^{2(K+1)}}{2\left(1-\rho^{2(K+1)}\right)^{3 / 2}} \frac{|x| \sqrt{1-\rho^{2}}}{\sigma} .
\end{aligned}
$$

Next, note that, for $x \leq(1-\rho)^{-1 / 2}, Z(x, \rho) \geq \Phi(-\sqrt{2} / \sigma)>0$, so

$$
\begin{aligned}
& \sup _{x<(1-\rho)^{-1 / 2}}\left|\frac{Z(x, \rho)-Z(x, \rho, K)}{Z(x, \rho)}\right| \\
& \quad \leq C \sup _{x<(1-\rho)^{-1 / 2}}\left(\rho^{2(K+1)} \phi\left(\frac{|x| \sqrt{1-\rho^{2}}}{\sigma}\right) \frac{|x| \sqrt{1-\rho^{2}}}{\sigma}+\frac{\bar{F}\left(S_{K}(\rho)^{1 / 2}\right)}{1-\rho}\right) \\
& \quad \leq C^{\prime}\left(\rho^{2(K+1)}+\frac{\bar{F}\left((1-\rho)^{-1 / 2}\right)}{1-\rho}\right)
\end{aligned}
$$

for some constants $C, C^{\prime}>0$; in the second inequality we used the facts that $\phi(|z|)|z|$ is 
bounded and $S_{K}(\rho)^{1 / 2} \sim 1 / \sqrt{2(1-\rho)}$. Since $\rho^{K} \rightarrow 0$ and $z^{2} \bar{F}(z) \rightarrow 0$ as $z \rightarrow \infty$, then the supremum above converges to 0 . To analyze the supremum over $\left[(1-\rho)^{-1 / 2}, \infty\right)$, note that

$$
\begin{aligned}
& \sup _{x \geq(1-\rho)^{-1 / 2}}\left|\frac{Z(x, \rho)-Z(x, \rho, K)}{Z(x, \rho)}\right| \\
& \quad \leq \sup _{x \geq(1-\rho)^{-1 / 2}} \frac{C^{\prime \prime}}{Z(x, \rho)}\left(\rho^{2(K+1)} \phi\left(\frac{|x| \sqrt{1-\rho^{2}}}{\sigma}\right) \frac{|x| \sqrt{1-\rho^{2}}}{\sigma}+\frac{\rho^{\alpha(K+1)} \bar{F}(x)}{1-\rho^{\alpha}}\right) \\
& \quad \leq \sup _{x \geq(1-\rho)^{-1 / 2}} \frac{C^{\prime \prime} \rho^{2(K+1)}}{\left(1-\rho^{\alpha}\right)^{-1} \bar{F}(x)} \phi\left(\frac{|x| \sqrt{1-\rho^{2}}}{\sigma}\right) \frac{|x| \sqrt{1-\rho^{2}}}{\sigma}+C^{\prime \prime} \rho^{\alpha(K+1)}
\end{aligned}
$$

for some other constant $C^{\prime \prime}>0$. Since $\rho^{\alpha K} \rightarrow 0$, it only remains to verify that the last supremum converges to 0 as well. To do this, fix $0<\delta<1$ and note that $1 / \bar{F}(x)=o\left(x^{\alpha+\delta}\right)$ as $x \rightarrow \infty$, so

$$
\begin{aligned}
& \sup _{x \geq(1-\rho)^{-1 / 2}} \frac{\rho^{2(K+1)}}{\left(1-\rho^{\alpha}\right)^{-1} \bar{F}(x)} \phi\left(\frac{|x| \sqrt{1-\rho^{2}}}{\sigma}\right) \frac{|x| \sqrt{1-\rho^{2}}}{\sigma} \\
& \quad=o\left(\rho^{2 K}(1-\rho)^{3 / 2} \sup _{x \geq(1-\rho)^{-1 / 2}} x^{1+\alpha+\delta} \exp \left(-\frac{x^{2}\left(1-\rho^{2}\right)}{2 \sigma^{2}}\right)\right) \\
& =o\left(\rho^{2 K}(1-\rho)^{(2-\alpha-\delta) / 2} \sup _{z \geq(1+\rho)^{1 / 2}} z^{1+\alpha+\delta} \exp \left(-\frac{z^{2}}{2 \sigma^{2}}\right)\right) \\
& =o\left(\exp \left(-2 K(1-\rho)+\frac{(\alpha+\delta-2)}{2}|\log (1-\rho)|\right)\right) \\
& =o(1) .
\end{aligned}
$$

This completes the proof.

\section{Acknowledgements}

The author would like to thank Peter Glynn for his insightful advice in the framing of this problem and helpful comments about the connections of these results to other areas. She would also like to thank the anonymous reviewer for thoroughly going through the proofs and suggesting ways to improve the paper.

\section{References}

[1] Barbe, P. AND McCormick, W. P. (2009). Asymptotic expansions for infinite weighted convolutions of heavy tail distributions and applications. Mem. Amer. Math. Soc. 197, 1-117.

[2] BASAK, G. K. AND Ho, K.-W. R. (2004). Level-crossing probabilities and first-passage times for linear processes. Adv. Appl. Prob. 36, 643-666.

[3] Billingsley, P. (1995). Probability and Measure. John Wiley, New York.

[4] Bingham, N. H., Goldie, C. M. And Teugels, J. L. (1987). Regular Variation. Cambridge University Press.

[5] Borovkov, A. A. And Borovkov, K. A. (2008). Asymptotic Analysis of Random Walks. Cambridge University Press.

[6] Chan, N. H. ANd WeI, C. Z. (1987). Asymptotic inference for nearly nonstationary AR(1) processes. Ann. Statist. 15, 1050-1063.

[7] Chen, Y., NG, K. N. AND TANG, Q. (2005). Weighted sums of subexponential random variables and their maxima. Adv. Appl. Prob. 37, 510-522.

[8] CLINe, D. (1983). Estimation and linear prediction for regression, autoregression and arma with infinite variance data. Doctoral Thesis, Colorado State University. 
[9] Cline, D. (1983). Infinite series of random variables with regularly varying tails. Tech. Rep. 83-24, Institute of Applied Mathematics and Statistics, University of British Columbia.

[10] Cox, D. D. (1991). Gaussian likelihood estimation for nearly nonstationary AR(1) processes. Ann. Statist. 19, 1129-1142.

[11] Cumberland, W. G. And Sykes, Z. M. (1982). Weak convergence of an autoregressive process used in modeling population growth. J. Appl. Prob. 19, 450-455.

[12] Davis, R. AND Resnick, S. (1988). Extremes of moving averages of random variables from the domain of attraction of the double exponential distribution. Stoch. Process. Appl. 30, 41-68.

[13] Finster, M. (1982). The maximum term and first passage times for autoregressions. Ann. Prob. 10, 737-744.

[14] GeluK, J. L. AND De VRIES, C. G. (2006). Weighted sums of subexponential random variables and asymptotic dependence between returns on reinsurance equities. Insurance Math. Econom. 38, 39-56.

[15] Jessen, A. H. And Mikosch, T. (2006). Regularly varying functions. Publ. Inst. Math. 80, 171-192.

[16] Mikosch, T. and Samorodnitsky, G. (2000). The supremum of a negative drift random walk with dependent heavy-tailed steps. Ann. Appl. Prob. 10, 1025-1064.

[17] NoviKov, A. (1990). On the first passage time of an autoregressive process over a level and an application to a 'disorder' problem. Theory Prob. Appl. 35, 269-279.

[18] Olvera-Cravioto, M. and Glynn, P. (2009). On the transition from heavy traffic to heavy tails for the M/G/1 queue: the semiexponential case. In preparation.

[19] Olvera-Cravioto, M., Blanchet, J. and Glynn, P. (2009). On the transition from heavy traffic to heavy tails for the M/G/1 queue: the regularly varying case. Submitted.

[20] Petrov, V. V. (1995). Limit Theorems of Probability Theory. Oxford University Press.

[21] Resnick, S. (1987). Extreme Values, Regular Variation, and Point Processes. Springer, New York.

[22] Rootzén, H. (1986). Extreme value theory for moving average processes. Ann. Prob. 14, 612-652.

[23] RozovskIǏ, L. V. (1989). Probabilities of large deviations of sums of independent random variables with a common distribution function that belongs to the domain of attraction of a normal law. Theory Prob. Appl. 34, 625-644.

[24] Van der Meer, T., Pap, G. And van Zuijlen, M. C. A. (1999). Asymptotic inference for nearly unstable AR( $p$ ) processes. Econometric Theory 15, 184-217.

[25] ZeEvi, A. And Glynn, P. W. (2004). Recurrence properties of autoregressive processes with super-heavy-tailed innovations. J. Appl. Prob. 41, 639-653. 